\title{
The Influence of Transient Thermal Gradients and Substrate Constraint on Delamination of Thermal Barrier Coatings
}

\section{Citation}

Sundaram, S., D. M. Lipkin, C. A. Johnson, and John W. Hutchinson. 2013. The influence of transient thermal gradients and substrate constraint on delamination of thermal barrier coatings. Journal of Applied Mechanics 80(1): 011002.

\section{Published Version}

doi:10.1115/1.4007727

\section{Permanent link}

http://nrs.harvard.edu/urn-3:HUL.InstRepos:10196729

\section{Terms of Use}

This article was downloaded from Harvard University's DASH repository, and is made available under the terms and conditions applicable to Open Access Policy Articles, as set forth at http:// nrs.harvard.edu/urn-3:HUL.InstRepos:dash.current.terms-of-use\#OAP

\section{Share Your Story}

The Harvard community has made this article openly available.

Please share how this access benefits you. Submit a story.

\section{Accessibility}




\title{
The Influence of Transient Thermal Gradients and Substrate Constraint on Delamination of Thermal Barrier Coatings
}

\author{
S. Sundaram, D. M. Lipkin, C. A. Johnson \\ GE Global Research Center, Niskayuma, NY 12309 \\ J. W. Hutchinson* \\ School of Engineering and Applied Sciences, Harvard University, \\ Cambridge, MA 02138
}

\begin{abstract}
A systematic study of factors affecting the delamination energy release rate and mode mix of a thermal barrier coating attached to a substrate is presented accounting for the influence of thermal gradients combined with rapid hot surface cooling. Transient thermal gradients induce stress gradients through the coating and substrate which produce overall bending if the substrate is not very thick and if it is not constrained. Due to their influences on the coating stresses, substrate thickness and constraint are important aspects of the mechanics of delamination of coating-substrate systems which must be considered when laboratory tests are designed and for lifetime assessment under in-service conditions. Temperature gradients in the hot state combined with rapid cooling give rise to a maximum energy release rate for delamination that occurs in the early stage of cooling and that can be considerably larger than the driving force for delamination in the cold state. The rates of cooling that give rise to a large early stage energy release rate are identified.
\end{abstract}

Keywords: Thermal barrier coatings, delamination, temperature gradients, substrate bending, bending constraint

*Corresponding author: hutchinson@husm.harvard.edu

\section{Introduction}

In service, thermal barrier coatings (TBCs) are subject to significant thermal gradients and occasional events involving rapid cooling of the hot surface. Above certain limits, yet to be established, these conditions are believed to promote coating delamination. Increasingly, efforts are made to replicate these conditions in the laboratory. The combination of thermal gradients and rapid cooling produces a transient, non-uniform stress distribution through the coating-substrate multilayer. Under such 
conditions, the largest driving force for delamination can occur shortly after the onset of cooling rather than in the cold state as is often assumed. In addition, the stress distribution depends on the extent the layered system undergoes overall bending, which, in turn, is a function of the thickness of the substrate and the manner in which it is constrained. It will be shown that substrate thickness and constraint significantly affect the driving force for delamination, and it will be argued that they must be considered in evaluating coating delamination, both in service and in laboratory tests.

The aims of this paper are two-fold: (i) To realistically characterize transient temperature and stress distributions in thermal barrier coating systems under rapid cooldown situations. (ii) To determine the transient energy release rate and mode mix for coating delaminations as dependent on the initial thermal gradient, the rate of cool-down, the thickness of the substrate and the extent to which the substrate is constrained against bending. The paper builds on earlier work of Evans and Hutchinson [1] wherein simplified representations of transient cooling were considered for coatings on thick substrates that allowed no bending. To set the stage for the present study, that work will be summarized at the end of this Introduction. First, however, the properties controlling the distributions of temperature and stress in the coating system will be defined.

Although many TBCs have three or more layers, the system considered in this paper is a bilayer comprised of a single coating bonded to a substrate. The bilayer can illustrate the essential points related to the aims of the paper stated above. The discussion is targeted to coatings used in aircraft and power generating turbines, but the findings are more widely applicable. For quantitative delamination results applicable to systems with more layers, it will be necessary to carry out calculations specific to those systems.

The bilayer is shown in Fig. 1. The substrate is layer \#1 with Young's modulus, Poisson's ratio and coefficient of thermal expansion (CTE) denoted by $E_{1}, v_{1}$ and $\alpha_{1}$. Its thermal conductivity and diffusivity are denoted by $k_{1}$ and $\kappa_{1}$. The corresponding quantities for the coating (layer \#2) are $E_{2}, v_{2}, \alpha_{2}, k_{2}$ and $\kappa_{2}$. Temperaturedependence of these quantities can be taken into account in the analyses given below, however, for simplicity these quantities will be taken to be independent of temperature without sacrificing the aims of the paper. 
At any instant of time, $t$, the temperature of the gas above the coating is denoted by $T_{2}^{g a s}(t)$, while that below the substrate is denoted by $T_{1}^{g a s}(t)$. Denote the temperature at the top surface of the coating by $T_{2}^{\text {sur }}(t)$, that at the interface by $T^{\text {int }}(t)$, and that at the bottom surface of the substrate by $T_{1}^{\text {sur }}(t)$. The heat transfer coefficient, $H_{2}$, relates the instantaneous heat flux, $q\left(\mathrm{wm}^{-2}\right)$, into the top surface of the coating according to $q=H_{2}\left(T_{2}^{\text {gas }}-T_{2}^{\text {sur }}\right)$. Similarly, the heat flux out of the bottom surface of the substrate is $q=H_{1}\left(T_{1}^{\text {sur }}-T_{1}^{\text {gas }}\right)$. Under steady-state conditions with $T_{1}^{\text {gas }}$ and $T_{2}^{\text {gas }}$ prescribed to be independent of time, the uniform heat flux and temperature distribution are

$$
\begin{aligned}
& q=\left(T_{2}^{\text {gas }}-T_{1}^{\text {gas }}\right)\left(\sum_{i=1}^{2}\left(\frac{1}{H_{i}}+\frac{h_{i}}{k_{i}}\right)\right)^{-1} \\
& T_{1}^{\text {sur }}=T_{1}^{\text {gas }}+q / H_{1}, T_{2}^{\text {sur }}=T_{2}^{\text {gas }}-q / H_{2} \\
& T^{\text {int }}=\left(\frac{k_{1}}{h_{1}} T_{1}^{\text {sur }}+\frac{k_{2}}{h_{2}} T_{2}^{\text {sur }}\right)\left(\frac{k_{1}}{h_{1}}+\frac{k_{2}}{h_{2}}\right)^{-1}
\end{aligned}
$$

with linear variations of $T$ through each of the two layers. Cases will also be considered where the hot state steady-state conditions are set by specifying the surface temperatures, $T_{1}^{\text {sur }}$ and $T_{2}^{\text {sur }}$. Then, (1) applies with $H_{1} \rightarrow \infty, H_{2} \rightarrow \infty, T_{1}^{\text {gas }} \rightarrow T_{1}^{\text {sur }}$ and $T_{2}^{\text {gas }} \rightarrow T_{2}^{\text {sur }}$.

In all cases considered in this paper, the initial temperature distribution, $T^{0}(y)$, will be the "hot" condition specified by the steady-state distribution (1). This distribution will either be specified by $T_{1}^{\text {gas }}$ and $T_{2}^{\text {gas }}$ along with the respective heat transfer coefficients, or, alternatively, by specifying $T_{1}^{\text {sur }}(0)$ and $T_{2}^{\text {sur }}(0)$. Calculations of the stress distribution and the energy release rate and the mode mix of delamination cracks will assume that the stresses in the coating are zero in the initial hot condition. This is a customary assumption for the coating attributed to the fact that creep is expected to relax stress at the highest temperatures. In addition, the present study will not account for mechanical stressing of the system, but a brief discussion of such effects will be given in the Conclusions.

The paper will explore how variations of the most important bilayer parameters affect the delamination energy release rate and mode mix during cool-down. The roles of 
the initial thermal gradient, the rate of switching on the cooling gas, and changing the hot surface heat transfer conditions will also be examined. The reference scenario models a set of aggressive laboratory tests conducted on a plasma spray coating involving a large initial temperature gradient across the coating and rapid cooling of the coating surface. This reference will be referred to with the abbreviation, the JETS simulation. Details of the cooling history will be specified in the next section.

The analysis of the bilayer described above carried out in [1] was simplified in the following way. The substrate was taken to be very thick $\left(h_{1}>>h_{2}\right)$, allowing no overall bending, and it was assumed to have a uniform temperature, $T^{\text {sub }}$. Consequently, the interface temperature was also assumed to be $T^{\text {sub }}$. With the temperature of the surface of the coating denoted by $T_{2}^{\text {sur }}$, the distribution of $T$ through the coating was taken to vary linearly from $T_{2}^{\text {sur }}$ to $T^{\text {sub }}$ at the interface. The initial values of these temperatures in the hot state were taken as $T_{2}^{\text {sur }}(0)$ and $T^{\text {sub }}(0)$, and the stress in the coating was taken to be zero in this state. Under these simplifying assumptions, the energy release rate, $G$, of a delamination crack on the interface is the elastic energy in the coating which is released upon interface separation. During cool-down when the surface and substrate (and interface) temperatures are, $T_{2}^{\text {sur }}$ and $T^{\text {sub }}$, respectively, the energy release rate is

$$
\tilde{G} \equiv \frac{2 G\left(1-v_{2}\right)}{E_{2} h_{2}\left(1+v_{2}\right)}=\frac{1}{3}\left(\alpha_{2} \Delta T_{\text {sur } / \text { sub }}\right)^{2}-\left(\alpha_{2} \Delta T_{\text {sur } / \text { sub }}\right)\left(\Delta \alpha \Delta T_{\text {sub }}\right)+\left(\Delta \alpha \Delta T_{\text {sub }}\right)^{2}
$$

where $\Delta \alpha=\alpha_{1}-\alpha_{2}$ is the CTE mismatch and

$$
\begin{aligned}
& \Delta T_{\text {sub }}=T^{\text {sub }}(0)-T^{\text {sub }} \\
& \Delta T_{\text {sur } / \text { sub }}=\left(T^{\text {sur }}(0)-T^{\text {sur }}\right)-\left(T^{\text {sub }}(0)-T^{\text {sub }}\right)
\end{aligned}
$$

Here, $\Delta T_{\text {sub }}$ is the temperature drop of the substrate and $\Delta T_{\text {sur/sub }}$ is the temperature drop of the coating surface relative to that of the substrate. A formula for the relative proportion of the mode I and II stress intensity factors, $K_{I}$ and $K_{I I}$, as measured by $\psi=\tan ^{-1}\left(K_{I I} / K_{I}\right)$ will be presented later. The delamination crack is considered to be sufficiently long such that it has attained steady-state propagation conditions. 
The energy release rate (2) provides insights into the driving force for delamination. It can be re-written as

$$
\frac{1}{3}\left(\frac{\alpha_{2} \Delta T_{\text {sur } / \text { sub }}}{\sqrt{\tilde{G}}}\right)^{2}-\left(\frac{\alpha_{2} \Delta T_{\text {sur } / \text { sub }}}{\sqrt{\tilde{G}}}\right)\left(\frac{\Delta \alpha \Delta T_{\text {sub }}}{\sqrt{\tilde{G}}}\right)+\left(\frac{\Delta \alpha \Delta T_{\text {sub }}}{\sqrt{\tilde{G}}}\right)^{2}=1
$$

characterizing an ellipse (see Fig. 2) in terms of the normalized variables $\Delta \alpha \Delta T_{\text {sub }} / \sqrt{\tilde{G}}$, which reflects the thermal strain mismatch between the coating and substrate, and $\alpha_{2} \Delta T_{\text {sur } / \text { sub }} / \sqrt{\tilde{G}}$, which reflects rapid cooling of the coating relative to the substrate. The outer ellipse in Fig.2 has been plotted using results derived later; it applies to a representative bilayer that is not constrained against bending. While the ellipse for the constrained case (5) applies for any set of parameters, that for the unconstrained case applies only for the specific set of bilayer parameters referred to in the figure caption. Due to the inverse dependence on $\sqrt{\tilde{G}}$ in the normalized variables, the energy release rate is significantly greater for the constrained bilayer than for the unconstrained bilayer for all combinations of $\left(\Delta T_{\text {sub }}, \Delta T_{\text {sur/sub }}\right)$.

Curves of constant $\tilde{G}$ are plotted in Fig. 3 for the constrained bilayer. Aspects related to delamination uncovered in the subsequent sections can be illustrated qualitatively in connection with this two-part figure.

(1) If the bilayer has a uniform temperature in the hot state, $T_{2}^{\text {sur }}(0)=T^{\text {sub }}(0)$, the limit when the entire bilayer is cooled to a uniform temperature, $T^{\text {cold }}$, with $T_{2}^{\text {sur }}=T^{\text {sub }}=T^{\text {cold }}$, has $\Delta T_{\text {sub }}=T^{\text {sub }}(0)-T^{\text {cold }}$ and $\Delta T_{\text {sur } / \text { sub }}=0$. This end point is depicted on Fig. 3A. For slow cooling conditions, $\Delta T_{\text {sur } / \text { sub }}$ remains nearly zero and the cooling trajectory follows the horizontal axis in Fig. 3A. The maximum $G$ occurs in the cold state with

$$
\frac{2 G\left(1-v_{2}\right)}{E_{2} h_{2}\left(1+v_{2}\right)}=\left[\Delta \alpha\left(T^{\text {sub }}(0)-T^{\text {cold }}\right)\right]^{2}
$$

Much of the discussion of TBC delamination in the literature has tended to focus on slow cooling in the absence of a hot state thermal gradient. However, even in the absence of an initial thermal gradient, if rapid cool-down occurs, the surface of the coating will cool 
faster than the substrate such that $\Delta T_{\text {sur/sub }}$ can be quite large in the early stages of cooldown. As depicted in Fig. 3A for rapid cooling, it will be seen that the maximum $G$ is due to $\Delta T_{\text {sur/sub }}$ and it occurs relatively early after the onset of cooling.

(2) If the bilayer has an initial temperature gradient in the hot state and is cooled to a uniform temperature, $T^{\text {cold }}$, then in the cold state $\Delta T_{\text {sub }}=T^{\text {sub }}(0)-T^{\text {cold }}$ and $\Delta T_{\text {sur } / \text { sub }}=\left(T_{2}^{\text {sur }}(0)-T^{\text {sub }}(0)\right)$. This cold state end point is depicted in Fig. 3B. Due to the interaction between $\Delta T_{\text {sub }}$ and $\Delta T_{\text {sur/sub }}$, the energy release rate in the cold state in the presence of the initial temperature gradient is less than it would be if $T_{2}^{\text {sur }}(0)=T^{\text {sub }}(0)$. This surprising reduction can be understood if one notes that the stress produced by cooling the coating due to the hot state gradient is tensile ( $\propto E_{2} \alpha_{2} \Delta T_{\text {sur/sub }}$ ), while the stress in the cooled state due to the CTE mismatch ( $\left.\Delta \alpha=\alpha_{1}-\alpha_{2}>0\right)$ is compressive $\left(\propto-E_{2} \Delta \alpha \Delta T_{\text {sub }}\right)$. These contributions offset one another. It will be shown that for some representative cool-down scenarios, the maximum $G$ does not occur in the cold state but instead occurs early during the transient cool-down driven primarily by $\Delta T_{\text {sur/sub }}$, as depicted in Fig. 3B. Before the substrate has had a chance to cool, $\Delta T_{\text {sub }} \cong 0$ such that, by (2),

$$
\frac{2 G\left(1-v_{2}\right)}{E_{2} h_{2}\left(1+v_{2}\right)} \cong \frac{1}{3}\left[\alpha_{2} \Delta T_{\text {sur } / \text { sub }}\right]^{2}
$$

The tensile stress that develops in the coating during this early transient period depends on the full CTE of the coating, $\alpha_{2}$, not the smaller CTE mismatch, $\Delta \alpha$.

(3) The relative amount of the mode I and mode II stress intensity factors as measured by $\tan \psi=K_{I I} / K_{I}$ will be provided later in connection with specific examples. In anticipation of these results, the trends in mode mix experienced by the delamination crack are superimposed on the interaction plot in Fig. 2. In particular, cooling trajectories dominated by $\Delta T_{\text {sub }}$ produce compression in the coating and give rise to mode II, or nearmode II, delaminations. Those dominated by $\Delta T_{\text {sur/sub }}$ produce tension in the coating and near-mode I delaminations. Those involving combinations of $\Delta \alpha \Delta T_{\text {sub }}$ and $\alpha_{2} \Delta T_{\text {sur/sub }}$ produce mixed mode delaminations. 


\subsection{Representative Properties for the Reference Scenario}

The present extensions of the Evans-Hutchinson [1] analysis to finite thickness substrates subject to transient thermal loadings with heat transfer conditions at the bilayer surfaces have the disadvantage that the quantities of interest cannot be expressed in closed form formulas such as those above. Results require numerical analysis of specific cases, albeit quite simple numerical analyses. The following properties will be used to specify the primary scenario in this paper. These property values can be regarded as representative of a plasma spray coating on a superalloy substrate [2]:

$$
\begin{array}{cc}
\text { substrate: } & h_{1}=3.5 \mathrm{~mm}, \kappa_{1}=5 \times 10^{-6} \mathrm{~m}^{2} \mathrm{~s}^{-1}, k_{1}=20 \mathrm{Wm}^{-1} \mathrm{C}^{-1}, \\
& \alpha_{1}=13 \times 10^{-6} \mathrm{C}^{-1}, E_{1}=100 \mathrm{GPa}, v_{1}=0.38 \\
\text { coating: } & h_{2}=0.75 \mathrm{~mm}, \kappa_{2}=0.6 \times 10^{-6} \mathrm{~m}^{2} \mathrm{~s}^{-1}, k_{2}=1.5 \mathrm{Wm}^{-1} \mathrm{C}^{-1}, \\
& \alpha_{2}=11 \times 10^{-6} \mathrm{C}^{-1}, E_{2}=30 G P a, v_{2}=0.2
\end{array}
$$

For the reference JETS scenario the initial steady-state temperature distribution is specified by (1) using surface temperatures at $t=0$ (not initial gas temperatures):

$$
T_{2}^{\text {sur }}(0)=1425 C, T_{1}^{\text {sur }}(0)=870 C
$$

For $t>0$, rapid cooling is imposed by abruptly switching on cooling gas with

$$
\left.\begin{array}{l}
T_{2}^{\text {gas }}=38 C, T_{1}^{g a s}=38 C \\
H_{2}=1500 \mathrm{Wm}^{-2} \mathrm{~K}^{-1}, H_{1}=200 \mathrm{Wm}^{-2} \mathrm{~K}^{-1}
\end{array}\right\}
$$

The heat transfer coefficient of the substrate, $H_{1}$, is representative of radiative cooling and natural convection, while that for the coating surface, $\mathrm{H}_{2}$ is representative of forced air cooling.

A number of the properties listed above for the reference scenario will be varied to reveal their influence. In addition, the effect of switching on the cooling gas and changing the hot surface heat transfer coefficient in a finite period of time will also be illustrated.

\section{Transient Thermal Analysis of Bilayer with Finite Thickness Substrate}

With the initial temperature distribution in the bilayer denoted by $T^{0}(y)$, the transient distribution for $t>0, T(y, t)$, is determined by the following boundary value 
problem. Let $T_{1}^{g a s}(t)$ and $T_{2}^{\text {gas }}(t)$ be specified with $T(y, 0)=T^{0}(y)$. On the bottom and top surfaces:

$$
\left.\begin{array}{l}
k_{1} \frac{\partial T(0, t)}{\partial y}=-H_{1}\left(T_{1}^{g a s}(t)-T(0, t)\right) \\
k_{2} \frac{\partial T\left(h_{1}+h_{2}, t\right)}{\partial y}=H_{2}\left(T_{2}^{g a s}(t)-T\left(h_{1}+h_{2}, t\right)\right)
\end{array}\right\}
$$

On the interface:

$$
k_{1} \frac{\partial T\left(h_{1}^{-}, t\right)}{\partial y}=k_{2} \frac{\partial T\left(h_{1}^{+}, t\right)}{\partial y}, T\left(h_{1}^{-}, t\right)=T\left(h_{1}^{+}, t\right)
$$

In the lower and upper layers:

$$
\left.\begin{array}{ll}
\frac{\partial T}{\partial t}=\kappa_{1} \frac{\partial^{2} T}{\partial^{2} x}, & 0 \leq y \leq h_{1} \\
\frac{\partial T}{\partial t}=\kappa_{2} \frac{\partial^{2} T}{\partial^{2} x}, & h_{1} \leq y \leq h_{1}+h_{2}
\end{array}\right\}
$$

This is a one-dimensional, transient heat conduction problem which must be solved numerically, except for special cases. In this paper, a standard finite difference method has been used to generate the temperature distributions and the thermal stresses derived from them. The time scale associated with thermal transients in a representative substrate (8) having thickness, $h_{1}=3.5 \mathrm{~mm}$, is $\tau_{1}=h_{1}^{2} / \kappa_{1}=2.4 \mathrm{~s}$ and about three times that of the coating with thickness $h_{2}=0.75 \mathrm{~mm}, \tau_{2}=h_{2}{ }^{2} / \kappa_{2}=0.9 \mathrm{~s}$.

An example illustrating the evolution of the transient temperature distribution for the bilayer with properties specified in (8) is presented in Fig. 4 for the JETS scenario with rapid cool-down (10). The initial temperature difference in hot state between the surface of the coating and the interface is approximately $400 \mathrm{C}$. The temperature at the surface of the coating drops by more than $600 \mathrm{C}$ within $0.2 \mathrm{~s}$ after the start of the cooldown process with the temperature at the interface and in the substrate having changed very little. The rapid cool-down phase takes place within the first second after the beginning of cooling. The large temperature drop of the coating relative to the substrate during this phase produces large tensile stress in the coating and a large energy release rate for delamination. In the JETS scenario, with cooling air at $38 \mathrm{C}$, it takes over $40 \mathrm{~s}$ for the bilayer to reach the cold state. 


\section{Stresses Induced by Cooling}

As noted previously, the stress in coating in the hot state is taken to be zero. The substrate is assumed to remain elastic at all temperatures. In the hot state the stress in the unconstrained substrate is also zero, because the stress in the coating is zero and the temperature distribution is linear. The stress in the constrained substrate will not generally be zero in the hot state, but only its stress change relative to the hot state enters into the computation of $G$ and $\psi$. Thus, for each case, the calculation in this section focuses on the stress change in the bilayer from the hot state. Plane strain conditions are assumed for the bilayer, and two cases will be considered to illuminate the role of bending on the stress distribution and the energy release rate: (i) complete constraint again bending with no longitudinal constraint and (ii) no constraint (c.f., Fig. 1). In the analysis that follows, the stress change relative to the hot state at any time during cooldown is determined for the uncracked bilayer far ahead of the delamination crack tip and in the two separated layers far behind the crack tip.

Denote the thermal strain by $\varepsilon_{T}$ measured from the initial hot state temperature,

$T^{0}(y)$; for a temperature-independent CTE, $\alpha, \varepsilon_{T}=\alpha\left(T(y, t)-T^{0}(y)\right)$.

\subsection{Stresses Far Ahead of the Delamination}

In the intact bilayer far ahead of the delamination, the strain along the bottom, $y=0$, is denoted by $\varepsilon_{0}$. For the case of unconstrained bending, the curvature of the bilayer is denoted by $\mathrm{K}$ and taken positive when curved downward. For the constrained case, $\mathrm{K}=0$. The change in the stress component acting parallel to the layer, $\sigma \equiv \sigma_{11}$, relative to the hot state is

$$
\sigma=\bar{E}\left(\varepsilon_{0}+\mathrm{K} y+\varepsilon^{T}\right)
$$

with $\bar{E}=E /\left(1-v^{2}\right)$. For the unconstrained bilayer, the requirements of overall equilibrium, i.e.,

$$
\int_{0}^{h_{1}+h_{2}} \sigma y^{j-1} d y=0, \quad j=1,2,
$$

provide the equations for $\varepsilon_{0}$ and $\mathrm{K}$, which are listed in the Appendix. For the bilayer constrained against bending, only the first of (15), $j=1$, applies with $\mathrm{K}=0$. 
Transient stress variations for the JETS scenario are presented in Fig. 5 for constrained and unconstrained bending at four locations in the intact bilayer far ahead of the crack tip. As will emerge clearly in sequel, the dominant contribution to the energy release rate of the delamination crack is the elastic energy in the coating. ${ }^{1}$ The largest coating stress occurs within the first second after the beginning of cooling. The stresses then slowly approach the asymptotic values associated with the cold state. A significant effect of the bending constraint on the stress distribution is seen. The stress in the coating at the surface is larger for the constrained case, as is the stress above the interface. Note that the peak tensile stress at the surface of the coating occurs at $t \cong 1 \mathrm{~s}$ just as the temperature in the substrate begins to undergo significant change (Fig. 4). Even though the substrate is more than 4 times as thick and 3 times as stiff as the coating, substrate bending gives rise to a significant reduction in the stresses in the coating. The difference in the stress distributions, with and without bending constraint, gives rise to large differences in the energy release rates, as will be seen.

\subsection{Stresses Far Behind the Delamination}

The stress change in each of the two separated layers far behind the delamination tip in Fig. 1 are also given by (14), where now $\varepsilon_{0 i}$ and $\mathrm{K}_{i}(i=1,2)$ must be determined for the individual layers using equilibrium or constraint conditions.

For the unconstrained case, first anticipate that the two layers are separated i.e., $\mathrm{K}_{1} \geq \mathrm{K}_{2}$, and require that each layer satisfy force and moment equilibrium:

$$
\int_{0}^{h_{1}} \sigma y^{j-1} d y=0, j=1,2, \text { and } \int_{h_{1}}^{h_{1}+h_{2}} \sigma y^{j-1} d y=0, j=1,2
$$

These provide $\varepsilon_{0 i}$ and $\mathrm{K}_{i}$ for the two layers (Appendix). In most of the examples in this paper, it will turn out that $\mathrm{K}_{1}>\mathrm{K}_{2}$, owing to the temperature gradient in the hot state. However, if the resulting solution gives $\mathrm{K}_{1}<\mathrm{K}_{2}$, the layers make contact. Then, as an approximation, a common curvature, $\mathrm{K}_{1}=\mathrm{K}_{2} \equiv \mathrm{K}$, is assumed. If friction between the

\footnotetext{
${ }^{1}$ The elastic energy in a thermally grown oxide layer in a multilayer TBC can contribute significantly to the energy release rate when the plane of delamination lies under the oxide layer and it would have to be accounted for in a multilayer simulation.
} 
contacting surfaces is neglected, the three equations of equilibrium providing $\varepsilon_{0 i}$ and $\mathrm{K}$ are

$$
\int_{0}^{h_{1}} \sigma d y=0, \int_{h_{1}}^{h_{1}+h_{2}} \sigma d y=0 \text { and } \int_{0}^{h_{1}+h_{2}} \sigma y d y=0
$$

For the constrained case $\left(\mathrm{K}_{1}=0\right)$, begin by anticipating that delamination separates the two layers $\left(\mathrm{K}_{2} \leq 0\right)$. The equations determining $\varepsilon_{0 i}(i=1,2)$ and $\mathrm{K}_{2}$ are

$$
\int_{0}^{h_{1}} \sigma d y=0, \int_{h_{1}}^{h_{1}+h_{2}} \sigma d y=0 \text { and } \int_{h_{1}}^{h_{1}+h_{2}} \sigma y d y=0
$$

If the result from (18) gives $K_{2}>0$, contact occurs; then, set $K_{2}=0$ and use the first two equations in (18) to determine $\varepsilon_{0 i}(i=1,2)$.

\section{Energy Release Rate and Mode Mix for Steady-state Interface Delamination}

The distribution of stress change from the hot state at any instant in the bilayer far ahead and far behind the delamination crack tip can be used to compute $G$ and $\psi$. The result for steady-state delamination applies to sufficiently long cracks emanating from either an edge or an open vertical crack in the coating. The length of the crack required for attaining steady-state depends on the details of the geometry of the emanating crack, but, typically, it must be longer than one coating thickness [3]. The steady-state computation makes use of energy changes from ahead to behind the crack tip evaluated at the same instant of time. In so doing, the computation assumes that the crack advances in a time period that is short compared to the time scale of the temperature changes, as would be the case for dynamic crack events.

The elastic energy/area of bilayer in Fig. 1 computed using the stress changes from the hot state given above is

$$
\begin{aligned}
& U_{A H E A D}=\frac{1}{2} \int_{0}^{h_{1}} \frac{\sigma^{2}}{\bar{E}_{1}} d y+\frac{1}{2} \int_{h_{1}}^{h_{1}+h_{2}} \frac{\sigma^{2}}{\bar{E}_{2}} d y \quad \text { (far ahead of delamination) } \\
& U_{B E H I N D}=\frac{1}{2} \int_{0}^{h_{1}} \frac{\sigma^{2}}{\bar{E}_{1}} d y+\frac{1}{2} \int_{h_{1}}^{h_{1}+h_{2}} \frac{\sigma^{2}}{\bar{E}_{2}} d y \quad \text { (far behind delamination) }
\end{aligned}
$$

\footnotetext{
2 Even with contact, the crack can be open at the tip with non-zero mode I component; an example is given in [4]. If frictional effects are not large, the energy release rate given by the procedure laid out here usually provides a good approximation and the crack is dominated by mode II.
} 
The energy release rate is given by

$$
G=U_{A H E A D}-U_{B E H I N D}
$$

A detailed derivation of this result will not be given here. One subtlety is the fact that $G$ and $\psi$ do not depend on the stress in the substrate in the hot state for the constrained case - they only depend on the change of stress in the substrate from the hot state. This result, which can be established using arguments similar to those given in [5], depends on the fact that the coating stress is taken to be zero in the hot state. The energy/area in the coating far ahead of the delamination,

$$
U_{\text {COATING }}=\frac{1}{2} \int_{h_{1}}^{h_{1}+h_{2}} \frac{\sigma^{2}}{\bar{E}_{2}} d y, \quad \text { (far ahead of delamination) }
$$

constitutes the main contribution to $G$ in most cases, as will be illustrated.

The mode mix, $\psi=\tan ^{-1}\left(K_{I I} / K_{I}\right)$, is obtained using solutions for a crack lying along an interface in a bilayer [5]. Consider the unconstrained case first. Define the force/thickness, $P$, and moments/thickness, $M$ and $M^{*}$, acting on the layers due the stress (12) in the uncracked bilayer (Fig. 6A) by

$$
\begin{aligned}
& P=-\int_{0}^{h_{1}} \sigma d y=\int_{h_{1}}^{h_{1}+h_{2}} \sigma d y \\
& M^{*}=\int_{0}^{h_{1}} \sigma\left(h_{1} / 2-y\right) d y, M=-\int_{h_{1}}^{h_{1}+h_{2}} \sigma\left(h_{1}+h_{2} / 2-y\right) d y
\end{aligned}
$$

This is an equilibrated set of forces and moments with $M^{*}=M+P\left(h_{1}+h_{2}\right) / 2$ and no traction acting on the interface. With these loads applied, a delamination crack can be introduced along the interface with no change in stress in the system. The stress intensity factors associated with delamination arise due to the removal of these loads to achieve the delaminated bilayer in Fig. 6C. Thus, the solution to the problem in Fig. 6B provides the stress intensity factors.

The complete solution [5] to the problem in Fig. 6B involves the elastic mismatch between the two layers which can be expressed in terms of the two Dundurs parameters (for plane strain):

$$
\alpha_{D}=\frac{\bar{E}_{2}-\bar{E}_{1}}{\bar{E}_{2}+\bar{E}_{1}}, \beta_{D}=\frac{1}{2} \frac{\mu_{2}\left(1-2 v_{1}\right)-\mu_{1}\left(1-2 v_{2}\right)}{\mu_{2}\left(1-v_{1}\right)+\mu_{1}\left(1-v_{2}\right)}
$$


with $\mu=E / 2(1+v)$ as the shear modulus. The parameter, $\beta_{D}$, is less important than $\alpha_{D}$ in the present problem, and it will be taken to be zero to simplify the formula for $\psi$ :

$$
\psi \equiv \tan ^{-1}\left(\frac{K_{I I}}{K_{I}}\right)=\tan ^{-1}\left[\frac{\lambda \sin \omega-\cos (\omega+\gamma)}{\lambda \cos \omega+\sin (\omega+\gamma)}\right]
$$

The following dimensionless parameters enter the evaluation of (25):

$$
\begin{aligned}
& \eta=h_{2} / h_{1}, \quad \Sigma=\bar{E}_{2} / \bar{E}_{1}, \quad A=\left[1+\Sigma\left(4 \eta+6 \eta^{2}+3 \eta^{3}\right)\right]^{-1}, I=\left[12\left(1+\Sigma \eta^{3}\right)\right]^{-1} \\
& \sin \gamma=6 \Sigma \eta^{2}(1+\eta) \sqrt{A I}, \quad \lambda=\sqrt{\frac{I}{A}} \frac{P h_{2}}{M}
\end{aligned}
$$

Elastic mismatch enters into $\psi$ through $\Sigma$ and $\omega\left(\alpha_{D}, \eta\right)$, which is tabulated in [5] $]^{3}$. The dependence of $\omega$ on elastic mismatch and $\eta$ is relatively weak for the problems under consideration here. For example, for the elastic mismatch specified by (8), $\alpha_{D}=-0.50$, and the tabulated values are $\omega=48.5^{\circ}$ for $\eta<<1$ and $\omega=45.6^{\circ}$ for $\eta=1$, while for no mismatch, $\alpha_{D}=0$, and $\omega=52.1^{\circ}$ for $\eta<<1$ and $\omega=49.1^{\circ}$ for $\eta=1$. Although the tabulated values of $\omega\left(\alpha_{D}, \eta\right)$ have been embedded and interpolated in the numerical code used to generate $\psi$ in this paper, a reasonable approximation for all the cases considered would be to take $\omega=52.1^{\circ}$, ignoring the mismatch and $\eta$-dependence of $\omega$.

No corresponding elasticity solution for the mode mix is available for the constrained bending problem. ${ }^{4}$ An infinitely deep substrate constrains bending, but it also constrains longitudinal straining of the substrate occurring during delamination. Of the two effects, the bending constraint is the more significant in determining the mode mix. As an approximation to the constrained bending case, the result (25) is used with $\eta \rightarrow 0$, corresponding to a coating delaminating from a deep substrate.

\section{Transient Delamination Energy Release Rate and Mode Mix}

Calculations of $G$ and $\psi$ during cool-down have been carried out for the JETS scenario introduced in Section 1.1. Results will be shown to illustrate the influence of

\footnotetext{
${ }^{3}$ The notation here follows that of [5], however, the numbering of the layers has been reversed.

${ }^{4}$ The discussion concerns only the mode mix. The expression for the energy release rate, (21), is exact.
} 
some of the most relevant system variables, including bending constraint, coating modulus, substrate thickness and thermal diffusivity, coating thickness, temperature gradients and cooling rate.

\subsection{The Role of Bending Constraint}

The results for the JETS scenario for the unconstrained and constrained cases are presented in Figs. 7 for the reference case with $h_{1}=3.5 \mathrm{~mm}$ and $h_{2}=0.75 \mathrm{~mm}$. Included with the delamination energy release rate and mode mix in these figures is the elastic energy/area stored in the coating ahead of the delamination, $U_{\text {COATING }}$ in (22). The following observations can be made.

(1) The maximum energy release rate occurs about one second following the onset of cool-down due to the rapid cooling of the coating surface and the associated large tensile stresses within the coating (c.f., Fig. 5). For both unconstrained and constrained cases, the delamination crack is dominantly mode I (i.e., $|\psi| \leq 20^{\circ}$ ) within this period. As the bilayer cools to the cold state at $t \approx 40 \mathrm{~s}$, the energy release rate decreases monotonically to a limit which is much lower than the maximum with an asymptotic mode mix that still has a dominant component of mode I.

(2) The maximum energy release rate for the bilayer constrained against bending is approximately twice that of the unconstrained case. This important effect is primarily due to the higher stresses in the coating for the bilayer constrained against bending (c.f., Fig.5).

(3) In most cases, $G$ is not more than $10 \%$ larger than $U_{\text {COATING }}$. These examples illustrate the fact that, even without bending constraint, the elastic energy in the coating provides the main contribution to the energy release rate. Much less energy is supplied from the substrate. Nevertheless, not all of the elastic energy in the coating is released upon separation unless the temperature distribution happens to be strictly linear at that instant.

\subsection{The Role of the Coating Modulus}

Fig. 8 shows the effect of the coating modulus, $E_{2}$, on the energy release rate for the unconstrained bilayer with no other changes in parameters of the JETS scenario. The 
dependence of $G$ on $E_{2}$ is strictly linear according to (2), and this holds to a good approximation for the unconstrained case as well. The energy in the coating ahead of the delamination, $U_{\text {COATING }}$, also scales linearly with $E_{2}$ when the substrate is stiff compared to the coating.

\subsection{The Role of the Substrate Thickness}

The reference bilayer has substrate thickness $h_{1}=3.5 \mathrm{~mm}$. The effect of thinner substrates the coating thickness is fixed at $h_{2}=0.75 \mathrm{~mm}$ is seen in Fig. 9. In these simulations the initial hot state temperatures of the interface and coating surface are fixed at the values associated with the JETS reference case, i.e., $T^{\text {int }}(0)=1013.9 C$ and $T_{2}^{\text {sur }}(0)=1425 \mathrm{C}$ corresponding to a fixed hot state heat flux, $q=0.822 \mathrm{MW} / \mathrm{m}^{2}$. Thinning the substrate reduces the energy release rate for both cases, however, the effect is most pronounced for the unconstrained substrate. When the substrate thickness ( $h_{1}=0.5 \mathrm{~mm}$ ) becomes less than the coating thickness, bending of the unconstrained substrate relieves much of the stress in the coating such that the energy release rate is greatly reduced.

\subsection{The Role of Substrate Thermal Diffusivity}

The examples discussed so far take $\kappa_{1}=5 \times 10^{-6} \mathrm{~m}^{2} \mathrm{~s}^{-1}$ as the thermal diffusivity of the substrate. The effect of four other choices for $\kappa_{1}$ on $G$ are illustrated in Fig. 10 for the otherwise unchanged JETS scenario for the case of unconstrained bending. The thermal diffusivity does not affect the initial steady-state temperature distribution, thus the primary influence of changing $\kappa_{1}$ is in altering the rate at which the substrate cools. A factor of 4 decrease or increase of $\kappa_{1}$ from the JETS value has a significant effect on the rate at which $G$ approaches the cold state limit, but it has somewhat less effect on the peak $G$ attained in the early stages of cool down. In particular, the change in the peak $G$ due to changing $\kappa_{1}$ by a factor of 4 is not nearly as large as the corresponding change resulting from constraining the substrate against bending seen in Fig. 7. The implication is that the reduction in the peak $G$ seen in Fig. 7, from constrained bending to unconstrained bending, and in Fig. 10, owing to change in substrate thermal diffusivity, is due to a combination of bending of the substrate imposed by the coating stress itself and 
changes in the temperature distribution within the substrate. This assertion is consistent with the evolution of temperature distribution in the substrate seen in Fig. 4 over the first several seconds after cool-down when the peak $G$ is attained.

\subsection{The Role of the Coating Thickness}

The effect of the coating thickness, $h_{2}$, on the delamination energy release for the unconstrained bilayer whose other properties are specified by (8) is presented in Fig. 11. In these simulations the substrate thickness is fixed at $h_{1}=3.5 \mathrm{~mm}$. Rapid cooling specified by (9) and (10) is imposed with the initial hot state temperatures of the interface and substrate surface fixed at the values associated with the JETS reference case: $T^{\mathrm{int}}(0)=1013.9 \mathrm{C}$ and $T_{1}^{\text {sur }}(0)=870 \mathrm{C}$, corresponding to a fixed hot state heat flux, $q=0.822 \mathrm{MW} / \mathrm{m}^{2}$. Two effects contribute to the dramatic reduction of the delamination energy release rate. First, for fixed hot state heat flux, the hot state temperature of the coating surface diminishes sharply with thinner coatings: $T_{2}^{\text {sur }}(0)=1425 C$ for $h_{2}=0.75 \mathrm{~mm} ; T_{2}^{\text {sur }}(0)=1260 C$ for $h_{2}=0.45 \mathrm{~mm}$; and $T_{2}^{\text {sur }}(0)=1096 C$ for $h_{2}=0.15 \mathrm{~mm}$. Secondly, even if the coating surface temperature in the hot state were fixed at $1425 C$, the elastic energy in the coating scales with the coating thickness. The reduction is most pronounced for the maximum $G$ which is attained early in the cool-down period. The cold state $G$ for the $0.15 \mathrm{~mm}$ and $0.45 \mathrm{~mm}$ coatings are nearly the same because the higher initial thermal gradient for the $0.45 \mathrm{~mm}$ coating counteracts the elastic mismatch contribution thereby lowering its cold state energy release rate, as discussed qualitatively in connection with Figs. 2 and 3.

\subsection{Effect of the Initial Thermal Gradient}

The effect of the initial temperature gradient across the unconstrained bilayer with reference properties (8) will be illustrated by considering various values of the initial hot surface temperature of the coating, $T_{2}^{\text {sur }}(0)$, in the otherwise unaltered JETS scenario, (9) and (10). For each simulation, the initial surface temperature of the substrate is $T_{1}^{\text {sur }}(0)=870 C$. In addition to the initial coating surface temperature, $T_{2}^{\text {sur }}(0)=1425 C$, used in the reference case, three other values of $T_{2}^{\text {sur }}(0)$ have been considered: $1240 C$, $1055 C$ and $870 C$ with the latter corresponding to a uniform initial temperature across 
the bilayer. These temperature differences across the bilayer have been chosen to illustrate the effect of the initial thermal gradient across the bilayer-they are not intended to be representative of thermal gradients under the highest heat flux. The transient behavior of $G$ and $\psi$ during cool-down is shown in Fig. 12. We emphasize again that in all the simulations, the stress is taken to be zero in the coating at the initial temperature distribution. The following points emerge.

(1) The smaller the initial temperature drop across the bilayer, the smaller the peak in $G$ that occurs within the first second after cooling starts. However, even with an initial temperature drop of $185 C$ across the bilayer $\left(T_{2}^{\text {sur }}(0)=1055 C\right)$, this peak in $G$ exceeds the asymptotic value of $G$ attained in the cold state. In the case of no initial thermal gradient $\left(T_{2}^{\text {sur }}(0)=870 C\right)$, the local maximum of $G$ in the early cool-down period is approximately the same as the cold state $G$. The mode difference between these two cases is again worth emphasizing: the peak $G$ in the early stages of cool-down is nearly mode I, while $G$ is the cold state is mode II or near-mode II.

(2) Fig. 12 illustrates that an initial temperature gradient across the bilayer usually lowers $G$ in the cold state. The largest cold state $G$ occurs for the bilayer with the uniform initial temperature, $T_{1}^{\text {sur }}(0)=T_{2}^{\text {sur }}(0)=870 \mathrm{C}$, even though it has the lowest initial temperature distribution.

(3) As noted earlier, in the early stages of cool-down (within the first few seconds) the delamination crack experiences near-mode I conditions. As cooling progresses, increasing mode II develops, as seen in Fig. 12B. For the two cases with the lowest initial temperature drop across the bilayer, the crack closes as cooling occurs and remains mode II for the remainder of cool-down.

Delamination in the cold state of a bilayer with $\alpha_{1}>\alpha_{2}$ will always be mode II if the initial temperature distribution is uniform and the coating stress is zero in the hot state.

\subsection{The Role of the Substrate Heat Transfer Coefficient}

Fig. 13 shows the effect of increasing the heat transfer coefficient at the substrate surface, $H_{1}$, on the delamination energy release rate and mode mix for the unconstrained 
bilayer (8) subject to the otherwise unaltered JETS scenario (9) and (10). The reference case with $H_{1}=200 \mathrm{Wm}^{-2} \mathrm{~K}^{-1}$ is included in the figure along with responses for $H_{1}=1000 \mathrm{Wm}^{-2} \mathrm{~K}^{-1}$ and $H_{1}=2000 \mathrm{Wm}^{-2} \mathrm{~K}^{-1}$. In all three simulations, the coating surface heat transfer coefficient is fixed at $H_{2}=1500 \mathrm{Wm}^{-2} \mathrm{~K}^{-1}$ and the initial hot state surface temperatures are $T_{1}^{\text {sur }}(0)=870 C$ and $T_{2}^{\text {sur }}(0)=1425 C$. The main effect of increasing $H_{1}$ is to decrease the time for the bilayer reach the cold state. The peak value of $G$ is hardly affected because it is caused by the rapid cooling of the coating surface and occurs before the substrate undergoes significant cooling. A ten-fold increase in $H_{1}$ has little effect on this peak energy release rate.

\subsection{The Role of the Coating Heat Transfer Coefficient}

Fig. 14 shows the effect of the heat transfer coefficient at the coating surface, $\mathrm{H}_{2}$, on the delamination energy release rate and mode mix for the unconstrained bilayer (8) subject to the otherwise unaltered JETS scenario (9) and (10). The reference case with $H_{2}=1500 \mathrm{Wm}^{-2} \mathrm{~K}^{-1}$ is included in the figure along with responses for lower, $H_{2}=250 \mathrm{Wm}^{-2} \mathrm{~K}^{-1}$, and higher, $H_{2}=9000 \mathrm{Wm}^{-2} \mathrm{~K}^{-1}$, coefficients, in all cases with the substrate surface coefficient maintained at $H_{1}=200 \mathrm{Wm}^{-2} \mathrm{~K}^{-1}$. The initial hot state surface temperatures are $T_{1}^{\text {sur }}(0)=870 C$ and $T_{2}^{\text {sur }}(0)=1425 C$ in all three simulations. The heat transfer coefficient of the coating surface has a significant effect on the peak $G$ in early stages of cool-down. The more rapid the cooling of the surface, the larger the surface temperature drop relative to the substrate, the larger the tensile stresses in the coating, and the larger the energy release rate.

\subsection{The Effect of a Delay in Switching on the Cooling Gas}

In all the simulations discussed above, the temperature of the cooling gas impinging on the substrate and coating surfaces is changed abruptly with zero transition time and the high rate of heat transfer at the coating surface is also switched on at the onset of cooling. In this subsection, the role of a time scale, $t_{0}$, in switching on the cooling gas will be investigated. In the next subsection, the effect of a delay in switching on a high rate of heat transfer at the coating surface will be analyzed. Neither of the two 
sets of simulations is intended to be an accurate representation of either a test set-up or an engine scenario, but they shed light on how the peak energy release rate is influenced by the rate at which cooling conditions arise. The reference bilayer (8) with layer thicknesses, $h_{1}=3.5 \mathrm{~mm}$ and $h_{2}=0.75 \mathrm{~mm}$, is used and unconstrained bending conditions are assumed.

Generally, the heat transfer coefficients change as well as the gas temperatures during the switch to cooling. In the JETS tests, the flame impinging on the coating surface is abruptly switched off but there is delay in moving the specimen into position to be subject to the cooling gas on the coating surface. Thus, for a short period of time the heat transfer coefficient on the coating surface remains unchanged at a relatively low level before increasing dramatically when the cooling gas begins to imping on it. First, however, to most clearly separate the several factors influencing the cooling rate, only the cooling gas temperatures will be changed. In these simulations, the heat transfer coefficients and initial gas temperatures are chosen to be consistent with the initial steady-state hot state temperature distribution (1), and the coefficients are held fixed as the gas temperature is lowered. The heat transfer coefficients used in these simulations are $H_{1}=988 \mathrm{Wm}^{-2} \mathrm{~K}^{-1}$ and $H_{2}=4698 \mathrm{Wm}^{-2} \mathrm{~K}^{-1}$ with initial hot state gas temperatures, $T_{1}^{\text {gas }}(0)=38 C$ and $T_{2}^{\text {gas }}(0)=1600 C$. From (1), these choices are consistent with the same initial steady-state hot state surface temperatures, $T_{1}^{\text {sur }}(0)=870 \mathrm{C}$ and $T_{2}^{\text {sur }}(0)=1425 \mathrm{C}$, and heat flux, $q=0.822 \mathrm{MW} / \mathrm{m}^{2}$, employed in the reference simulations described earlier. For $t \geq 0$, the gas temperature on the substrate surface is maintained at $T_{1}^{\text {gas }}=38 \mathrm{C}$ while the gas temperature on the coating surface is specified by

$$
T_{2}^{g a s}(t)=T_{2}^{g a s}(0) e^{-t / t_{0}}+T_{2}^{g a s}(\infty)\left(1-e^{-t / t_{0}}\right), \quad t \geq 0
$$

with $T_{2}^{\text {gas }}(\infty)=38 C$. Thus, $t_{0}$ is the exponential decay time for switching on the cooling gas on the coating.

The results based on the coating cooling gas history (26) are presented in Fig. 15 for various application times including abrupt cooling with $t_{0} \rightarrow 0$. Recall that the thermal time scale of the coating is $\tau_{2}=h_{2}{ }^{2} / \kappa_{2}=0.9 \mathrm{~s}$. 
(1) The sharp peak in $G$ that occurs within the first second under abrupt cooling is reduced significantly if the rate of applying the cooling gas is comparable to the time scale of the coating. The energy release rate in the fully cooled state at $T=38 C$ is not affected by the rate of application of the cooling gas. The peak $G$ for the abrupt limit, $t_{0}=0 s$, is greater than that for the reference JETS case primarily because the coating surface heat transfer coefficient is more than three times as large as that for the JETS case.

(2) The peak $G$ associated the slowest cooling rates $\left(t_{0}=2,4 s\right)$ shown in Fig. 15 are still much larger than the cold state energy release rate due to existence of the initial temperature gradient across the bilayer and the fact that the coating temperature drops more rapidly than the substrate (c.f., Fig. 3B). Thus, even when the cooling gas is switched on slowly there is a relatively sharp peak in $G$ in early stage of cool-down. The mode mix for these simulations is dominated by mode I.

In summary, the rate at which the cooling gas is switched on has an important effect on the peak energy release rate for delamination in the early stages of cool-down. The relevant time scale for this to be a factor is the thermal time scale of the coating, $\tau_{2}=h_{2}{ }^{2} / \kappa_{2}$. For a TBC coating with thickness on the order of a millimeter, the time scale is on the order of a fraction of a second. For a coating with thickness on the order of 100 microns, the time scale is two orders of magnitude smaller. Therefore, the transient behavior of $G$ associated with rapid switching of the cooling gas is unlikely to be relevant to the thinnest TBC coatings.

\subsection{The Effect of a Delay in Transitioning to High Heat Transfer at the Coating} Surface

Next, as introduced in the previous subsection, consider the effect of a delay in switching on a high level of heat transfer on the coating surface. Specifically, consider simulations which are identical to the JETS scenario with abrupt application of the cooling gas (at 38C) except that for $t \leq t_{0}, H_{2}=200 \mathrm{Wm}^{-2} \mathrm{~s}^{-1}$, and for $t>t_{0}$, $H_{2}=1500 \mathrm{Wm}^{-2} \mathrm{~s}^{-1}$. The effect of this delay on the peak $G$ seen in Fig. 16 is not nearly as significant as that produced by a slower rate of switching on the cooling gas. When 
the heat transfer coefficient jumps to $H_{2}=1500 \mathrm{Wm}^{-2} \mathrm{~s}^{-1}$, the sudden boost of coating cooling gives rise to a rapid increase in $G$ with a local peak that is not much reduced from the JETS case.

\section{Estimates of $G$ for Cracks Lying Above the Coating-substrate Interface}

As noted earlier, the elastic energy/area stored in the intact coating ahead of the delamination is usually a good approximation to $G$ for interface cracking. This statement applies equally well to a delamination crack paralleling the interface and lying within the coating at depth $h \leq h_{2}$ below the surface. Moreover, as the examples above have revealed, the crack is dominantly mode I at the peak energy release rate in the early stages of cool-down. These conditions create the possibility of cracks propagating parallel to the surface within the coating. The approximation,

$$
G \cong U=\frac{1}{2} \int_{h_{1}+h_{2}-h}^{h_{1}+h_{2}} \frac{\sigma^{2}}{\bar{E}_{2}} d y, \quad \text { (far ahead of delamination) }
$$

can be used to estimate $G$ for a crack within the coating a distance $h$ below the surface.

Fig. 17 for the unconstrained reference bilayer (8) subject to the reference JETS scenario presents the transient behavior of $U$ evaluated at three depths below the surface, including the full depth, $h=h_{2}$, at the interface. The energy stored in a surface layer with $2 / 3$ the coating thickness has a peak value which is only about $2 \%$ below that stored in the entire coating. More surprising, a surface layer with thickness $1 / 3$ that of the coating has a peak $U$ only $20 \%$ below that of the entire coating. While these results are specific to the case considered, they are not untypical. The high stresses near the surface of the coating in the early stages of cooling combined with the steep gradient of stress together concentrate the elastic energy toward the surface. Implications of these findings for intercoating delamination will be discussed in Conclusions.

\section{Conclusions}

The energy release rate for coatings delaminating from substrates constrained against bending can be significantly greater than for unconstrained substrates. The examples consider in this paper with representative TBC substrate thicknesses and a 
coating thickness on the order of $1 \mathrm{~mm}$ revealed that the maximum energy release rate for the constrained substrate is typically twice that for the unconstrained substrate. These findings suggest that substrate bending constraint should be taken into account in assessing coating delamination for engine components, and it is also an important consideration in evaluating laboratory tests. The standard coated circular substrate coupon is usually not constrained in thermal cycling tests, while a cylindrical tubular substrate that is coated and subject to burner rig testing is bending constrained.

The combination of a thermal gradient across the coating in the hot state and rapid cooling of the coating produces a driving force history for delamination distinctly different from that for relatively slow cooling under isothermal conditions. In particular, a coating with an initial thermal gradient subject to rapid cooling leads to a large peak in the delamination energy release rate at an early stage during cool-down. This peak occurs before the substrate temperature undergoes appreciable change, and thus it is due primarily to tensile stress in the coating proportional to $\alpha_{2} \Delta T_{\text {sur } / \text { sub }}$. By contrast, the stress in the uniform cold state temperature is a superposition of tensile stress due to initial thermal gradient and compressive stress proportional to CTE mismatch, $\Delta \alpha \Delta T_{\text {sub }}$. As illustrated in the paper, these stresses of differing sign usually offset one another to reduce the energy release rate in the cold state.

Coatings subject to slow cooling under isothermal conditions experience the maximum energy release (mode II) in the cold state, due to CTE mismatch. Numerous examples have been presented showing that rapid cool-down can give rise to an energy release rate in the early stage of cooling which is much larger than the cold state release rate, especially if the coating has an initial hot state thermal gradient. The mode mix of these early stage delaminations is dominantly mode I.

The rate of cooling considered rapid depends on the thermal time scale of the coating, $h_{2}{ }^{2} / \kappa_{2}$. For a $1 \mathrm{~mm}$ TBC coating, $h_{2}{ }^{2} / \kappa_{2} \approx 1 s$, while for a $100 \mu m$ coating it is $\approx 0.01 \mathrm{~s}$. For coating systems with realistic heat transfer conditions, it has been shown that switching on cooling gas in $0.5 \mathrm{~s}$ or less impinging on a $0.75 \mathrm{~mm}$ coating produces a large delamination energy release rate in the early stage of cool-down. Switching times of this order should be achievable in the laboratory, and it seems likely that extraordinary 
events in an engine might also lead to switching times this rapid. On the other hand, coatings whose thicknesses are on the order of $100 \mu \mathrm{m}$, such as those on aero engine blades, seem much less likely to experience cool-down times less than $h_{2}{ }^{2} / \kappa_{2}$ because that would require switching times on the order of 0.01s. Nevertheless, cooling that is slow compared to $h_{2}^{2} / \kappa_{2}$ but rapid compared to the larger substrate time scale, $h_{1}^{2} / \kappa_{1}$, will cool the coating to $\approx T^{\text {sub }}(0)$ before the substrate has time to cool. By (4), the temperature drop of the coating relative to the substrate at this relatively early stage is given by $\Delta T_{\text {sur } / \text { sub }} \cong T^{\text {sur }}(0)-T^{\text {sub }}(0)$ and, by (2) or (7),

$$
\frac{2 G\left(1-v_{2}\right)}{E_{2} h_{2}\left(1+v_{2}\right)} \cong \frac{1}{3}\left(\alpha_{2} \Delta T_{\text {sur } / \text { sub }}\right)^{2}
$$

This formula for constrained bending can be used to estimate the maximum $G$ during the early stage of cool-down for intermediate rates of cooling.

The findings in this paper rely heavily on the assumption that the stress in the coating stress relaxes to zero in the hot state. This assumption is commonly invoked based on the fact that the TBC coating creeps at high temperatures. Recent data [6,7] on plasma-sprayed $7 \mathrm{wt} \% \mathrm{Y}_{2} \mathrm{O}_{3}-\mathrm{ZrO}_{2}$ suggest that stresses in the range $20-80 \mathrm{MPa}$ will undergo significant creep relaxation within minutes for temperatures above $1000 C$. Presumably the rate of relaxation would be even great at higher stresses. This high rate of creep relaxation supports the working hypothesis that the stresses in the coating are nearly zero in the hot state, at least for portions of the coating above $1000 \mathrm{C}$. In addition to sidestepping uncertainty concerning residual stress, taking the coating to be zero in the hot state obviates the necessity of tracking the history of stress in the coating from one thermal cycle to another. Given the centrality of this assumption, further experiments to verify hot state stress relaxation in the coating should be carried out, including the temperature range $800-1000 \mathrm{C}$ relevant to coating material adjacent to the substrate.

The prediction of a large delamination energy release rate in the early stage of cool-down for thicker coatings raises other issues bearing on TBC material behavior. Under rapid to moderate-rate cooling, the largest energy release rate occurs early during cool-down when the coating and the interface are still quite hot $(\approx 800 C)$. Moreover, these are mode I dominated delaminations which can occur either along the interface or 
within the coating above the interface. The temperature dependence of the toughness of the coating and the interface will play a role in whether or not delaminations are triggered by the peak energy release rate. It seems reasonable to assume that the interface toughness may be significantly elevated by temperature due to the proximity of the interface to the bond coat. At $\approx 800 C$, bond coat plasticity is likely to relax stresses at the tip of an interface crack and increase dissipation accompanying delamination. However, the toughness of the ceramic coating material itself may be much less dependent on temperature. If so, the large early stage energy release rates may promote delamination within the coating away from the interface, as has been observed in laboratory experiments involving steep thermal gradients and rapid cooling [8].

Stress in the coating and the substrate due to mechanical loads has not been included. Under the assumption that stress in the coating in the hot state relaxes to zero, it can be shown that mechanical loads carried by the substrate will not influence the delamination energy release rate and mode mix if the mechanical loads are maintained constant while the temperature changes occur. Consequently, the peak energy release in the early stage of cool-down will usually not be altered by mechanical loads because they are not likely to change significantly during this period. The energy release rate in the cold state will be altered by the mechanical loads if they are different from those in the hot state.

Finally, as noted in the Introduction, delamination analysis of more complex TBCs, including the effect of a thermally grown oxide layer or a surface layer penetrated by calcium-magnesium-alumino-silicate (CMAS), requires these layers to be incorporated into the model. The elastic energy stored in the thermally grown oxide layer can comprise an appreciable fraction of the stored energy in thin TBC systems representative of those on aero engine blades. CMAS can significantly boost the driving force for delamination for any coating owing to its role in increasing the effective elastic modulus of the layer $[9,10]$ A computer code capable of the general analysis of multilayers is being developed by M. R. Begley [11]. 


\section{Appendix: Outline of the stress analysis and energy release rate calculations}

With the stress at any instant, $\sigma(y, t)$, given in terms of the thermal strain measured from the hot state, $\varepsilon^{T}(y, t)$, by (14), the strain change at the bottom of the substrate, $\varepsilon_{0}$, and curvature change, $\mathrm{K}$, of the intact unconstrained bilayer far ahead of the crack tip are given by

$$
\begin{aligned}
& A_{11} \varepsilon_{0}+A_{12} \mathrm{~K}=\int_{0}^{h_{1}+h_{2}} \bar{E} \varepsilon^{T} d y \equiv R_{1} \\
& A_{12} \varepsilon_{0}+A_{22} \mathrm{~K}=\int_{0}^{h_{1}+h_{2}} \bar{E} \varepsilon^{T} y d y \equiv R_{2}
\end{aligned}
$$

with $\bar{E}$ identified for each layer and

$$
\begin{gathered}
A_{11}=\bar{E}_{1} h_{1}+\bar{E}_{2} h_{2}, A_{12}=\frac{1}{2} \bar{E}_{1} h_{1}{ }^{2}+\frac{1}{2} \bar{E}_{2}\left(h_{2}{ }^{2}+2 h_{1} h_{2}\right), \\
A_{22}=\frac{1}{3} \bar{E}_{1} h_{1}^{3}+\frac{1}{3} \bar{E}_{2}\left(h_{2}^{3}+3 h_{2}{ }^{2} h_{1}+3 h_{1}{ }^{2} h_{2}\right)
\end{gathered}
$$

For the constrained bilayer $\varepsilon_{0}$ is given by the first of (A.1) with $K=0$. Far behind the crack tip the corresponding overall strain and curvature changes in the individual layers are given as follows. For the coating,

$$
\begin{aligned}
& A_{11} \varepsilon_{02}+A_{12} \mathrm{~K}_{2}=\int_{h_{1}}^{h_{1}+h_{2}} \bar{E}_{2} \varepsilon^{T} d y \\
& A_{12} \varepsilon_{02}+A_{22} \mathrm{~K}_{2}=\int_{h_{1}}^{h_{1}+h_{2}} \bar{E}_{2} \varepsilon^{T} y d y
\end{aligned}
$$

with

$$
A_{11}=\bar{E}_{2} h_{2}, A_{12}=\frac{1}{2} \bar{E}_{2}\left(h_{2}{ }^{2}+2 h_{1} h_{2}\right), A_{22}=\frac{1}{3} \bar{E}_{2}\left(h_{2}{ }^{3}+3 h_{2}{ }^{2} h_{1}+3 h_{1}{ }^{2} h_{2}\right)
$$

For the unconstrained substrate

$$
\begin{aligned}
& A_{11} \varepsilon_{01}+A_{12} \mathrm{~K}_{1}=\int_{0}^{h_{1}} \bar{E}_{1} \varepsilon^{T} d y \\
& A_{12} \varepsilon_{01}+A_{22} \mathrm{~K}_{1}=\int_{0}^{h_{1}} \bar{E}_{1} \varepsilon^{T} y d y
\end{aligned}
$$

with

$$
A_{11}=\bar{E}_{1} h_{1}, A_{12}=\frac{1}{2} \bar{E}_{1} h_{1}^{2}, A_{22}=\frac{1}{3} \bar{E}_{1} h_{1}^{3}
$$

For the constrained substrate the first of (A.5) gives $\varepsilon_{01}$ with $\mathrm{K}_{1}=0$. The procedure for dealing with cases for which the curvatures from the above recipes indicate contact across the delaminated interface is given in Section 3. 
The strain energy in the various layers can be directly evaluated. For example, for the intact bilayer ahead of the crack tip, (19) gives

$$
U_{\text {AHEAD }}=\frac{1}{2} A_{11} \varepsilon_{0}{ }^{2}+A_{12} \varepsilon_{0} \mathrm{~K}+A_{22} \mathrm{~K}^{2}-R_{1} \varepsilon_{0}-R_{2} \mathrm{~K}+\frac{1}{2} \int_{0}^{h_{1}+h_{2}} \bar{E}\left(\varepsilon^{T}\right)^{2} d y
$$

with analogous expressions for the other layers. These expressions are homogeneous of degree 2 in the temperature change from the hot state, and this gives rise to the elliptical curves of constant energy release rate in Fig. 2. The force and bending quantities defined in connection with the mode mix in (23) are readily evaluated using the stress from (A.1).

\section{Acknowledgements}

The authors are grateful to the following colleagues for their technical contributions and insights in support of this effort: W.C. Hasz (GE Global Research, Niskayuna, NY), Carlos G. Levi, and Erin M. Donohue (both from UC Santa Barbara). This work was supported in part by the U.S. Dept. of Energy under Cooperative Agreement DE-FC2605NT42643. Neither the United States Government nor any agency thereof, nor any of their employees, makes any warranty, express or implied, or assumes any legal liability or responsibility for the accuracy, completeness, or usefulness of any information, apparatus, product, or process disclosed, or represents that its use would not infringe privately owned rights. Reference herein to any specific commercial product, process, or service by trade name, trademark, manufacturer, or otherwise does not necessarily constitute or imply its endorsement, recommendation, or favoring by the United States Government or any agency thereof. The views and opinions of authors expressed herein do not necessarily state or reflect those of the United States Government or any agency thereof.

\section{References}

[1] Evans, A.G. and Hutchinson, J.W., 2007, "The mechanics of coating delamination in thermal gradients,” Surf. Coat. Technol., 201, pp. 7905-7916.

[2] Bunker, R.S., 2008, "The effects of manufacturing tolerances on gas turbine cooling," Proceedings of 2008 IGTI, ASME Turbo Expo: Power for Land Sea and Air. June 9-13, 2008, Berlin, Germany, pp. 1-9.

[3] Yu, H.H., He, M.Y. and Hutchinson, J.W., 2001, “ Edge effects in thin film delamination,” Acta Mater., 49, pp. 93-107. 
[4] Hutchinson, R.G. and Hutchinson, J.W., 2011, "Lifetime assessment for thermal barrier coatings: Tests for measuring mixed mode delamination toughness,” J. Am. Ceram. Soc., 94,[S1] pp. S85-S95.

[5] Suo, Z. and Hutchinson, J.W., 1990, “Interface crack between two elastic layers.”, Int. J. of Fracture, 43, pp. 1-18.

[6] Dickinson, G.R., Petorak, C., Bowman, K. and Trice, R.W., 2005, “Stress relaxation of compression loaded plasma-sprayed 7 wt\% Y2O3-ZrO2 stand-alone coatings,” J. Am. Ceram. Soc., 88, pp. 2202-2308.

[7] Petorak, C. and Trice, R.W., 2011, "Effect of heat-treatment on stress relaxation behavior of plasma-sprayed 7 wt\% Y2O3-ZrO2 stand-alone coatings,” Surface \& Coating Tech., 205, pp. 3218-3225.

[8] Lampenscherf, S., 2010, Private communication concerning laboratory tests conducted at Siemens Corporation.

[9] Mercer, C. , Faulhaber, S., Evans, A.G. and Darolia, R., 2005, “A delamination mechanism for thermal barrier coatings subject to calcium-magnesium-alumino-silicate (CMAS) infiltration,” Acta Mater. 53, pp. 1029-1039.

[10] Kramer, Yang, S. J., Levi, C.G. and Johnson, C. A., 2006, “Thermochemical interaction of thermal barrier coatings with molten $\mathrm{CaO}-\mathrm{MgO}-\mathrm{Al} 2 \mathrm{O} 3-\mathrm{SiO} 2$ (CMAS) deposits,” J. Am. Ceram. Soc., 89, pp. 3167-3175.

[11] Begley, M.R., 2012, "LayerSlayer: an open source code for analyzing multilayers” (under development). 


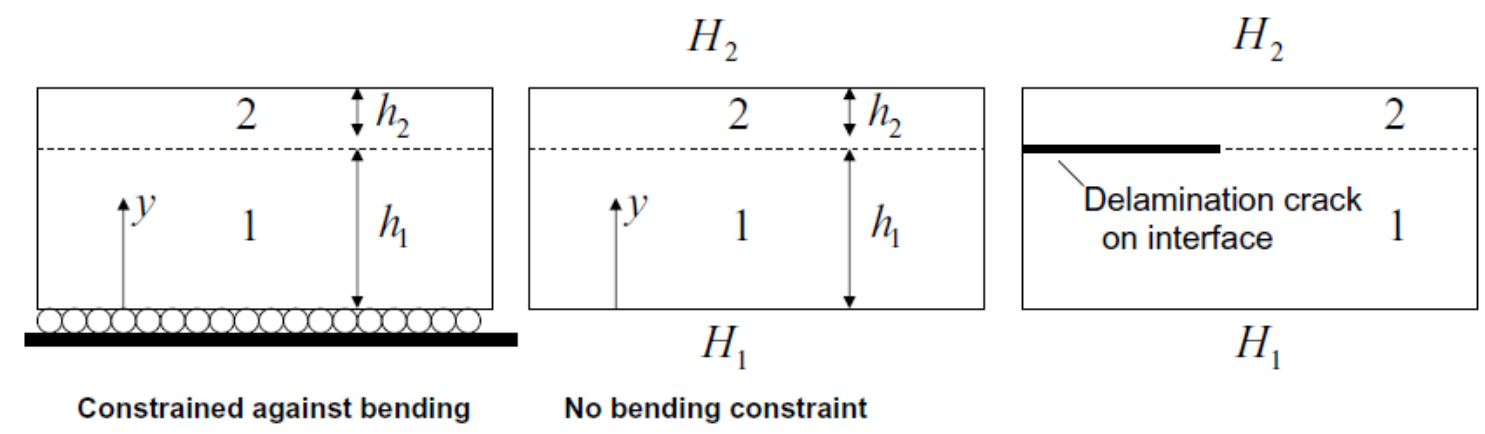

Fig. 1 The TBC bilayer with a coating on top of a substrate. Constrained and unconstrained conditions are depicted. The heat transfer coefficients are $H_{1}$ at the bottom surface and $H_{2}$ at the top surface. Delamination is analyzed for both the constrained and unconstrained cases. 


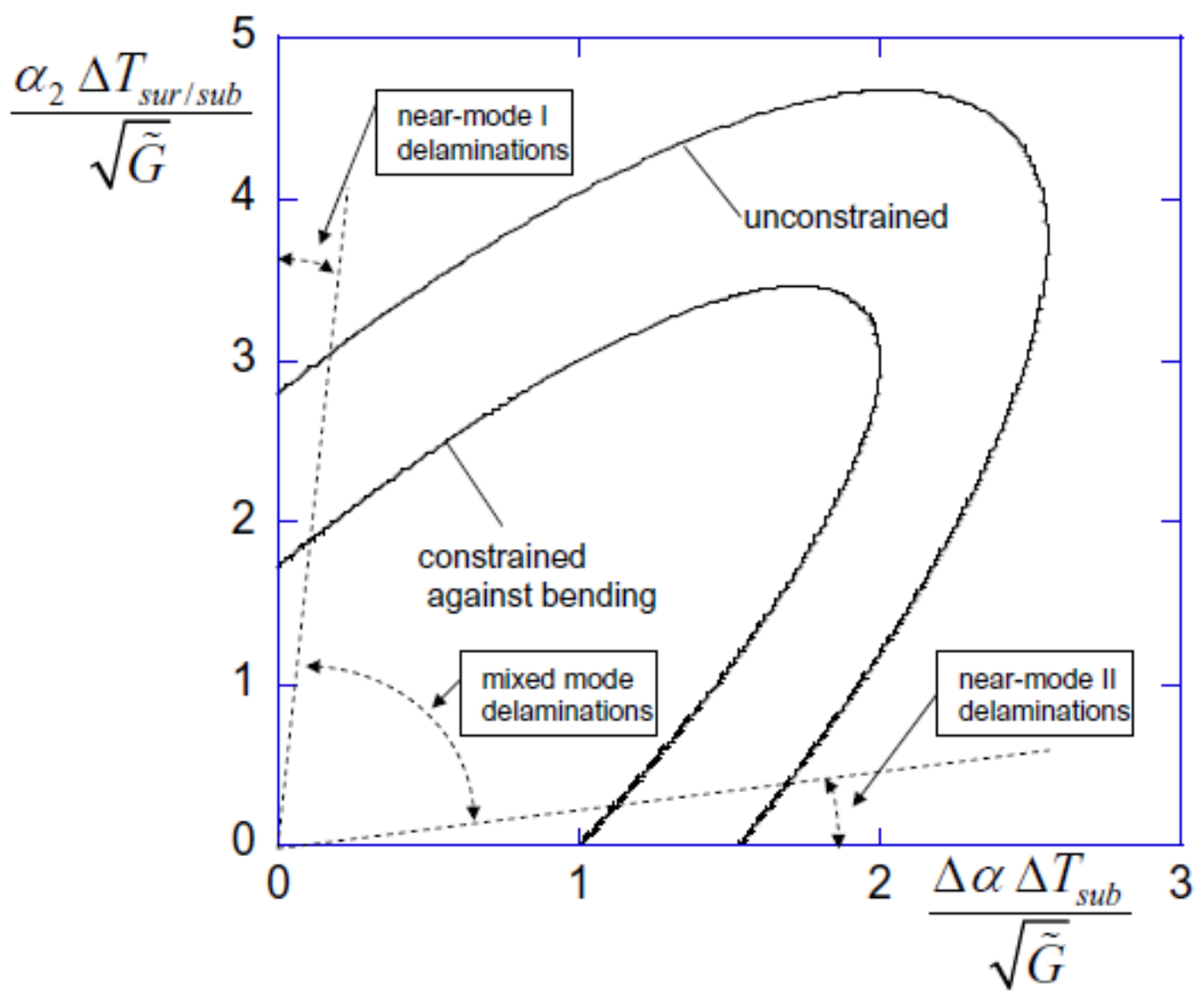

Fig. 2 Elliptical contours of constant delamination energy release rate with the normalized temperature drop of the substrate on the horizontal axis and the normalized temperature drop of the coating surface relative to the substrate on the vertical axis. The horizontal axis reflects the thermal strain mismatch between the coating and the substrate while the vertical axis reflects the effect of rapid cooling of the coating. Full details are given in the text. The curve for the constrained case applies to any set of bilayer parameters. The curve for the unconstained case applies only to a bilayer with parameters specified by (8). 

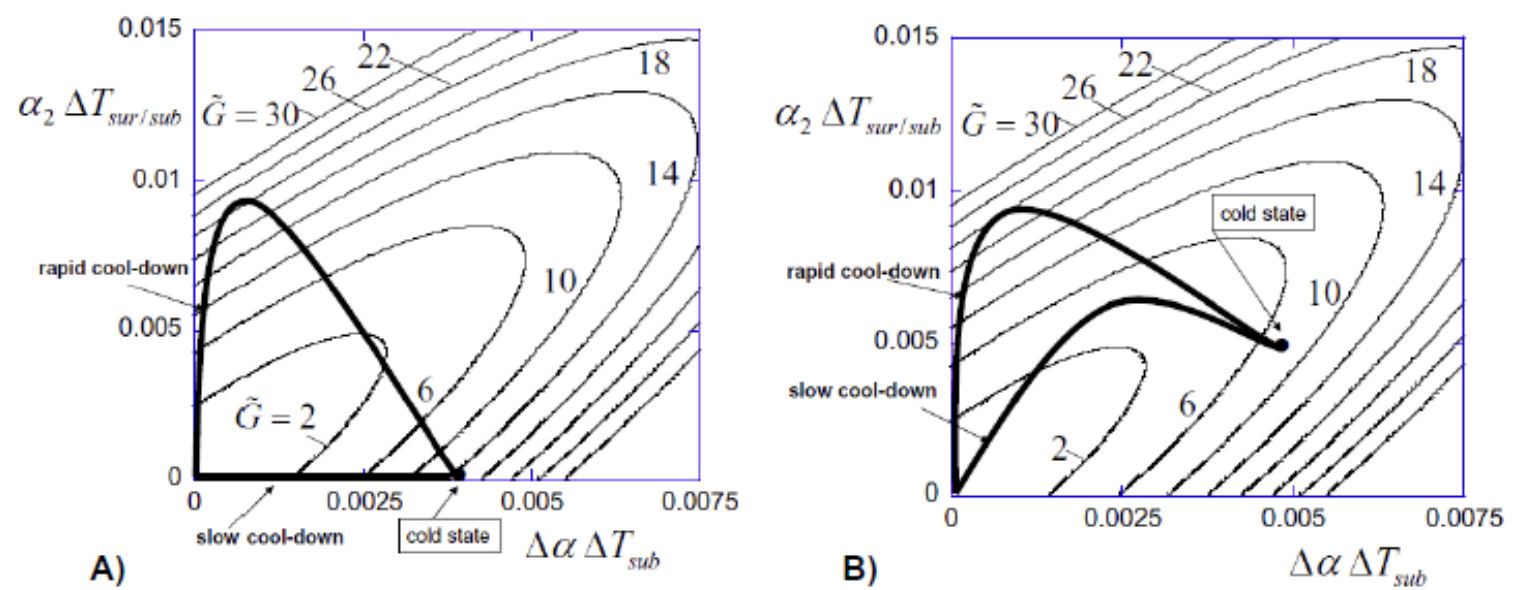

Fig. 3 Elliptical contours for constant delamination energy release rate for various $\tilde{G}=2 G\left(1-v_{2}\right) /\left(E_{2} h_{2}\left(1+v_{2}\right)\right)$ including depiction of rapid and slow cooling trajectories for a substrate constrained against bending. A) With no thermal gradient in the hot state. B) With a significant thermal gradient in the hot state. Even in the absence of a thermal gradient in the hot state a bilayer subject to rapid cooling of the coating can experience a large energy release rate driven by the temperature drop of the coating relative to the substrate before the substrate has had a chance to undergo much cooling. 


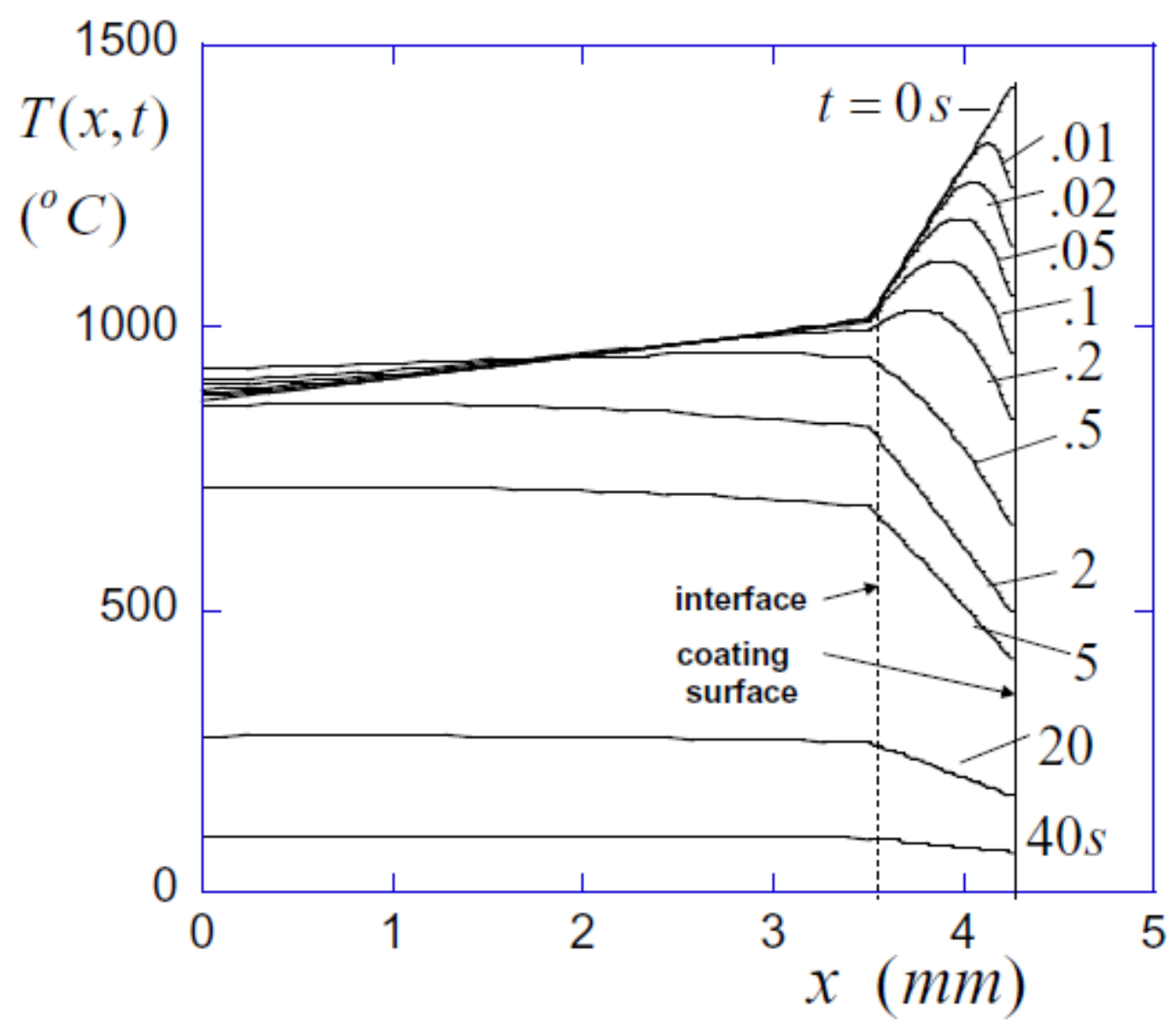

Fig. 4 Transient temperature distribution for a bilayer with properties (8) with $h_{1}=3.5 \mathrm{~mm}$ and $h_{2}=0.75 \mathrm{~mm}$ subject to the JETS scenario in (9) and (10). 

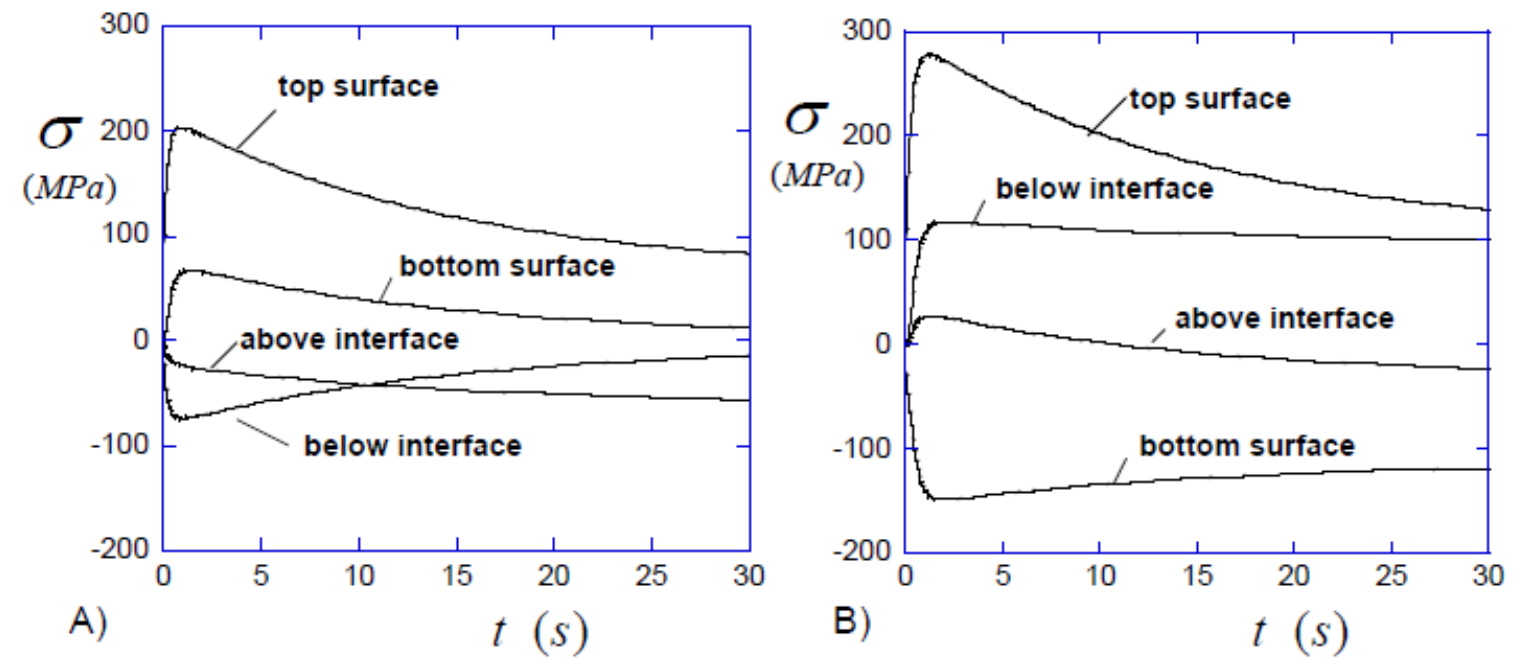

Fig. 5 Transient stress variations at four locations within the intact bilayer (well ahead of the delamination crack tip) with properties (8). The bilayer is subject to the JETS scenario specified by (9) and (10). A) No bending constraint. B) With bending constraint. The transient temperature distributions are those in Fig. 4. In all cases in this paper, the stress in the coating in the hot state is taken to be zero. The stress in the constrained substrate in the hot state does not contribute to the delamination energy release rate and it is taken to be zero in this figure. 


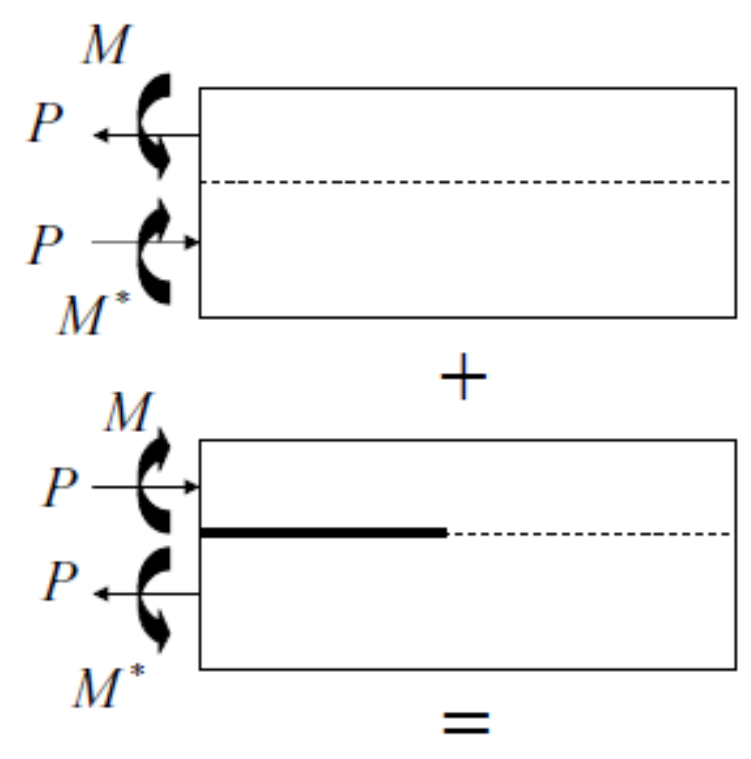

(A)

(C)

Fig. 6 The elasticity problem for computing the mode mix of the delamination crack, $\psi$, for the unconstrained bilayer subject to thermal stresses in (C). (A) The resultant forces and moments in each layer due to the thermal stress in the intact bilayer. (B) Equal and opposite resultant forces and moments that cancel the loads in (A) and that produce the stress intensity factors for problem (C). 

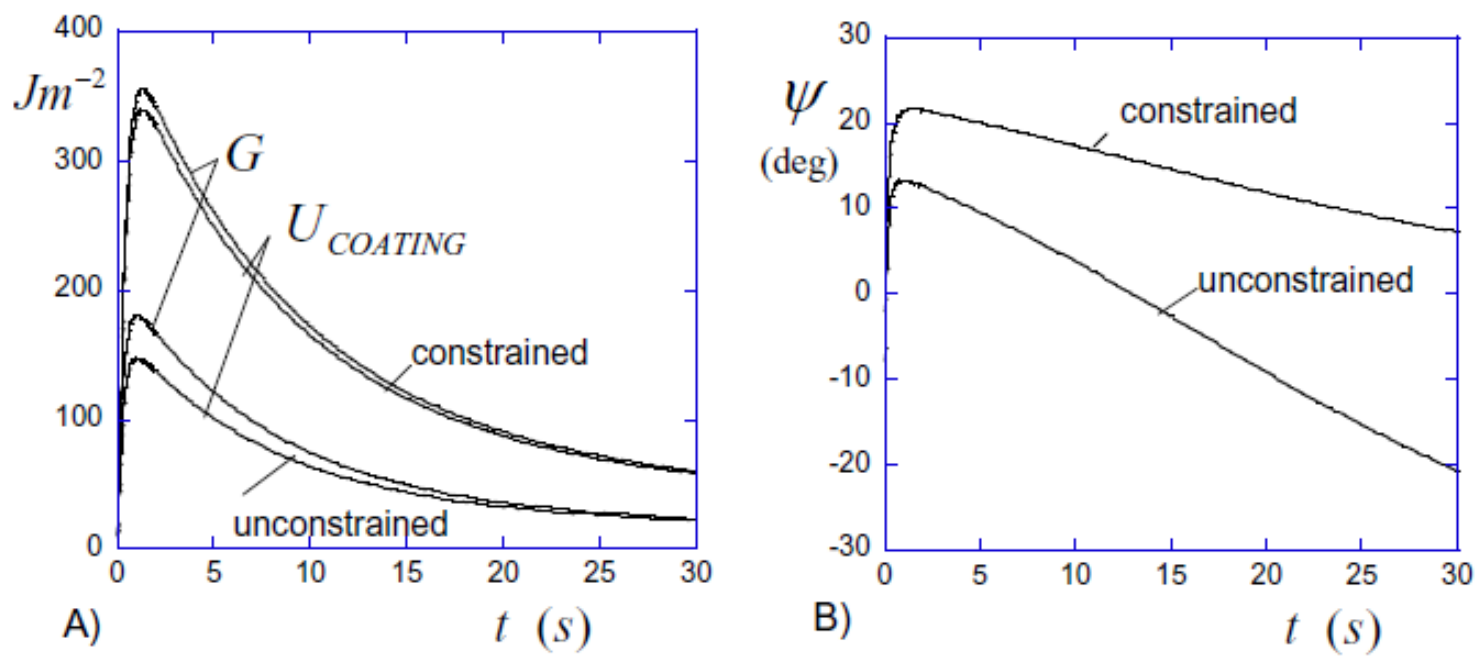

Fig. 7 (A) Transient variation of the delamination energy release rate and (B) the mode mix for a bilayer with properties (8) subject to the JETS scenario, (9) and (10). Results for both constrained and unconstrained bending are shown. The variations of the temperature and stress distributions are those in Figs. 4 and 5. Included for both cases is the variation of the energy/area in the coating, $U_{\text {COATING }}$, well ahead of the delamination crack tip. As is evident, $U_{\text {COATING }}$ supplies a good approximation to $G$. 


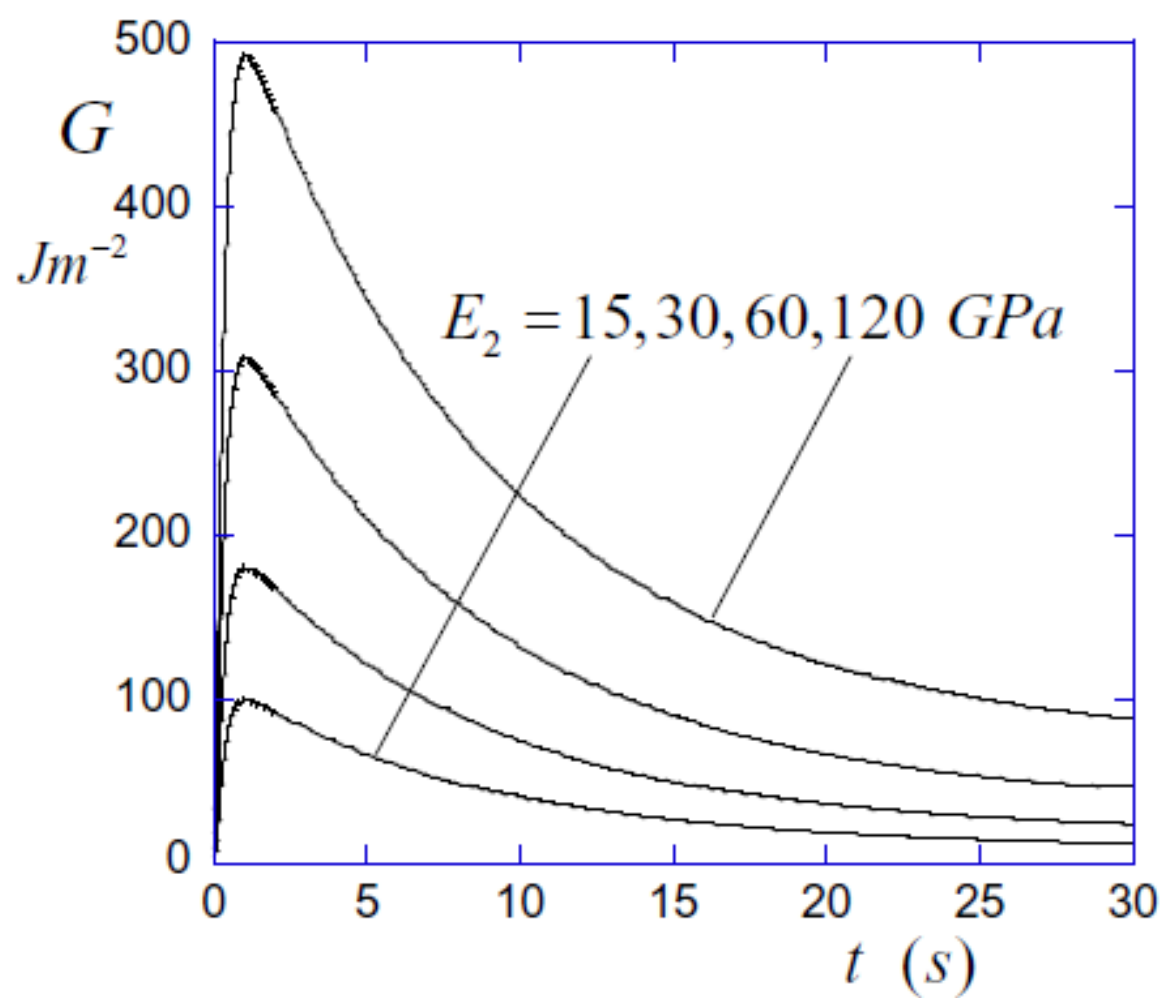

Fig. 8 The effect of the coating modulus, $E_{2}$, on the delamination energy release rate for an unconstrained bilayer specified by (8) and subject to the JETS scenario (9) and (10). 


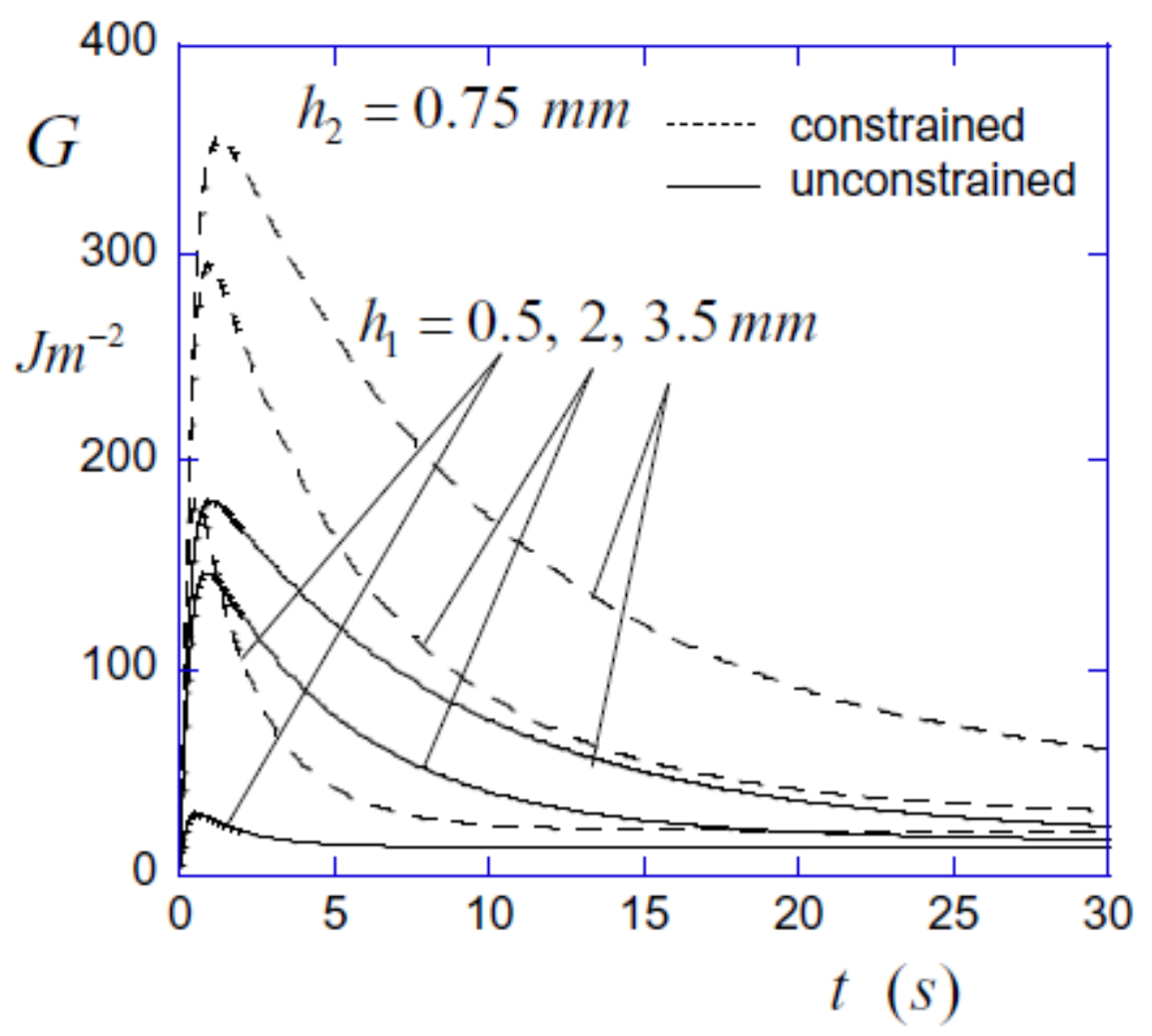

Fig. 9 The effect of the substrate thickness, $h_{1}$, on the delamination energy release for the bilayer whose other properties are specified by (8). The coating thickness is fixed at $h_{2}=0.75 \mathrm{~mm}$. Results for both constrained and unconstrained bending are shown for cooling given by (10). In all cases, the initial hot state temperatures of the interface and coating surface have the values associated with the reference JETS case:

$T^{\text {int }}(0)=1013.9 C$ and $T_{2}^{\text {sur }}(0)=1425 C$, corresponding to a fixed hot state heat flux, $q=0.822 \mathrm{MW} / \mathrm{m}^{2}$. 


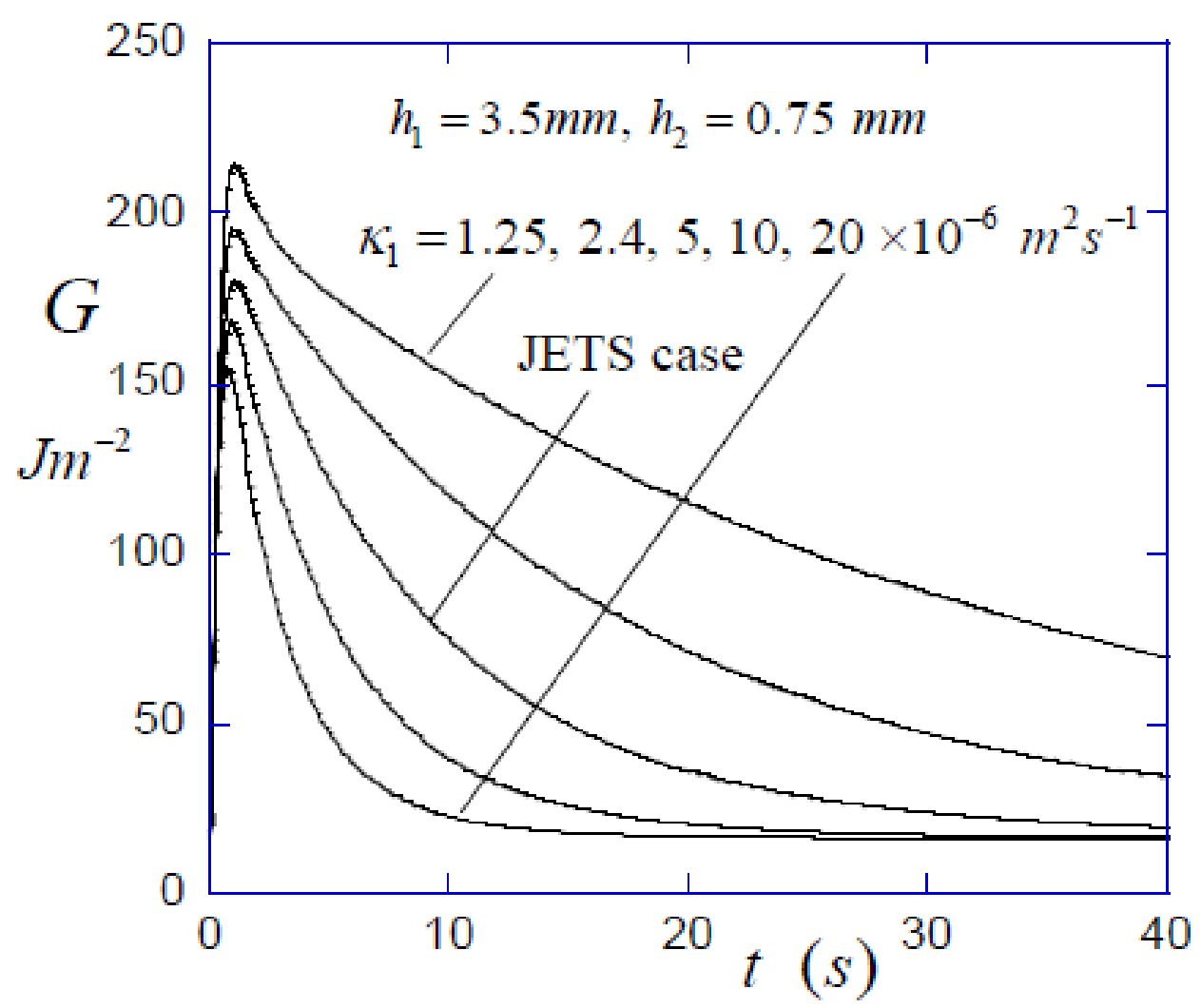

Fig. 10 The effect of varying the substrate thermal diffusivity, $\kappa_{1}$, on the delamination energy release for the unconstrained bilayer whose other properties are specified by (8). The initial steady-state hot state temperature distribution is unaffected by $\kappa_{1}$ and is the same as the reference JETS case with $T_{1}^{\text {sur }}(0)=800 C$ and $T_{2}^{\text {sur }}(0)=1425 C$, corresponding to a fixed hot state heat flux, $q=0.822 \mathrm{MW} / \mathrm{m}^{2}$. The substrate diffusivity has a significant effect on the cooling rate of the substrate and therefore on the rate of approach to $G$ in the cold state. The effect of substrate diffusivity on the peak $G$ in the early stages of cool-down is less pronounced. 


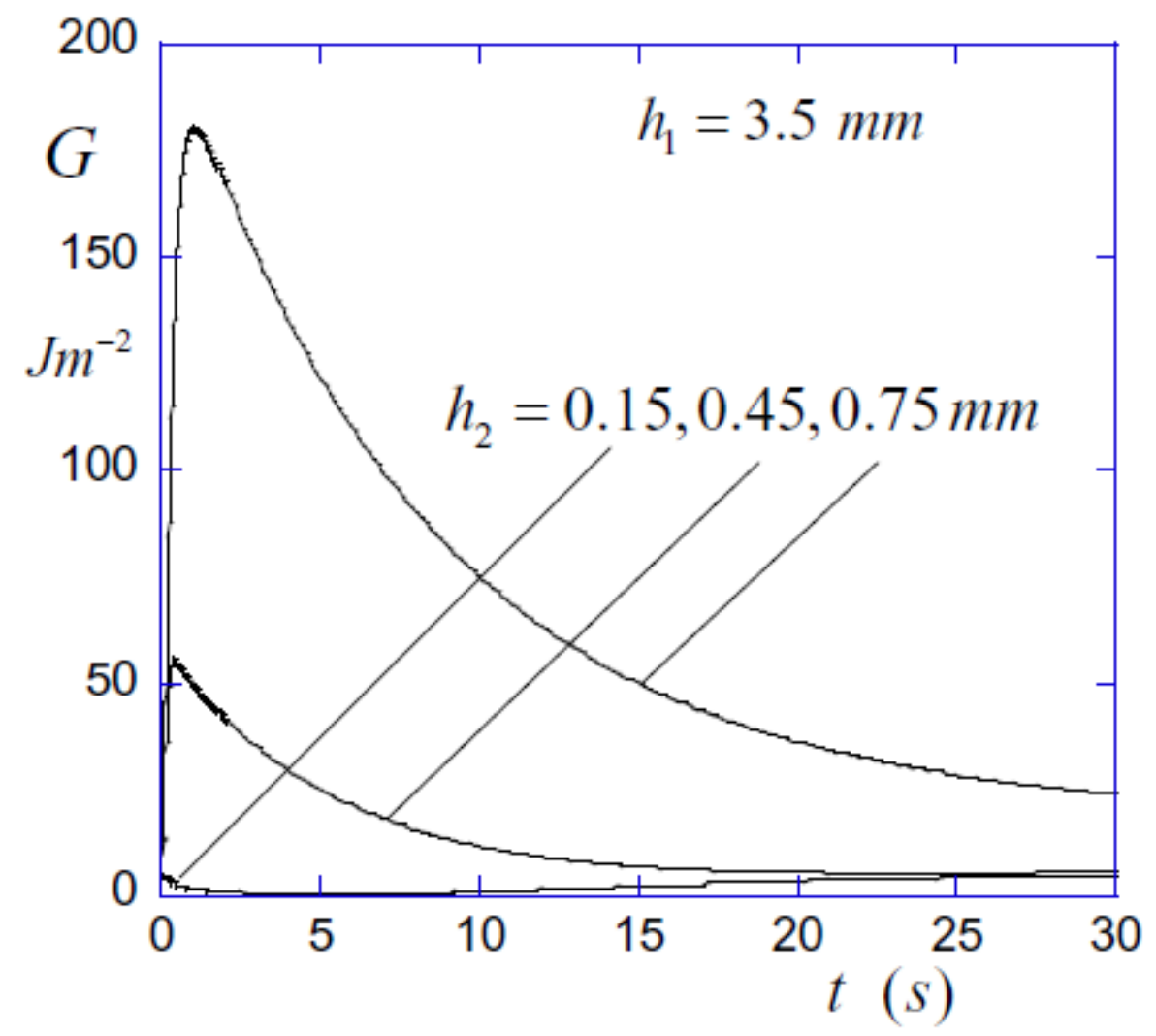

Fig. 11 The effect of the coating thickness, $h_{2}$, on the delamination energy release for the unconstrained bilayer whose other properties are specified by (8). The substrate thickness is fixed at $h_{1}=3.5 \mathrm{~mm}$. In all cases, the initial hot state temperatures of the interface and substrate surface are fixed at the values associated with the JETS reference case: $T^{\text {int }}(0)=1013.9 C$ and $T_{1}^{\text {sur }}(0)=870 C$, corresponding to a fixed hot state heat flux, $q=0.822 M W / m^{2}$. Cooling is specified by (10). 

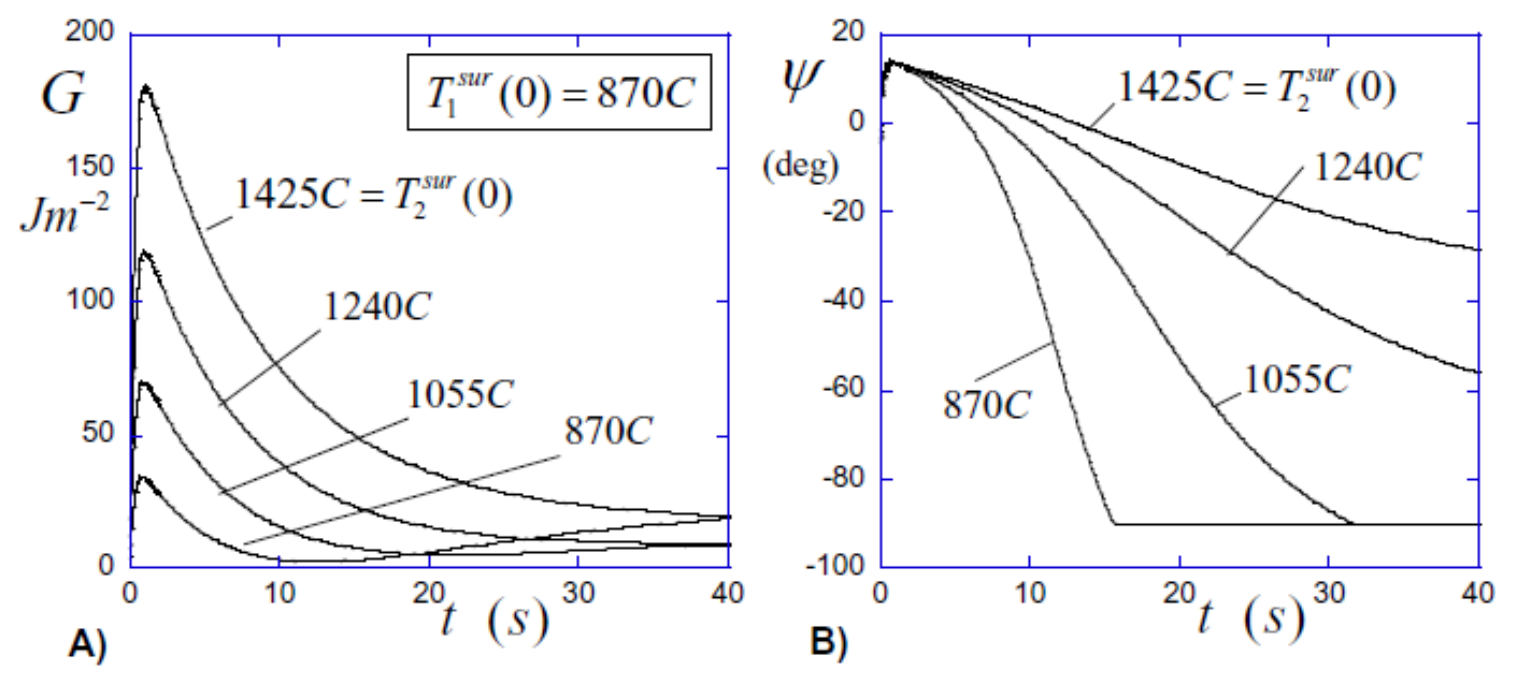

Fig. 12 The effect of the initial hot state coating surface temperature, $T_{2}^{\text {sur }}(0)$, on the delamination energy release rate and mode mix for the reference unconstrained bilayer (8) subject to JETS cooling (10). The initial temperature of the surface of the substrate is $T_{1}^{\text {sur }}(0)=870 \mathrm{C}$ for all the simulations . 

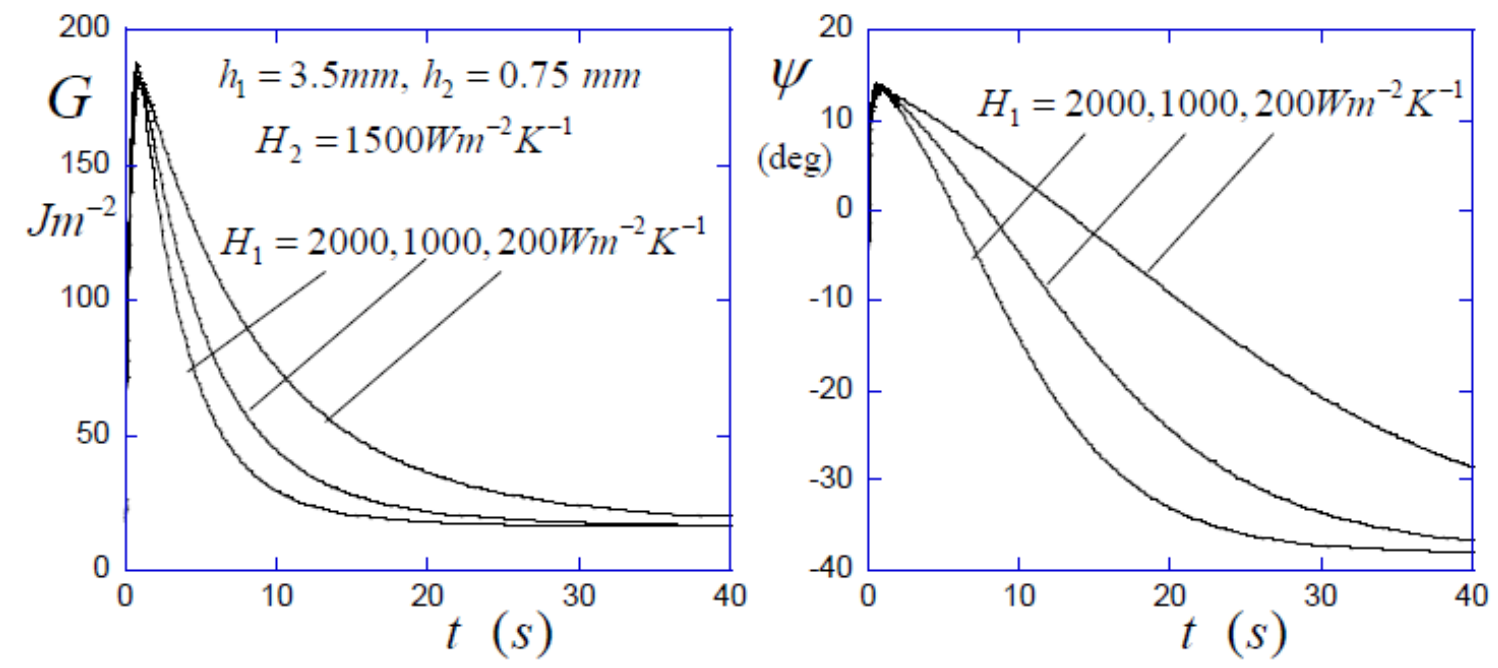

Fig. 13 Effect of increasing the heat transfer coefficient at the substrate surface, $H_{1}$, on the delamination energy release rate and mode mix for the unconstrained bilayer (8) subject to the otherwise unchanged cooling scenario (10). The JETS reference case has $H_{1}=200 \mathrm{Wm}^{-2} \mathrm{~K}^{-1}$ and $H_{2}=1500 \mathrm{Wm}^{-2} \mathrm{~K}^{-1}$. In all cases, $T_{1}^{\text {sur }}(0)=870 \mathrm{C}$ and $T_{2}^{\text {sur }}(0)=1425 C$ 

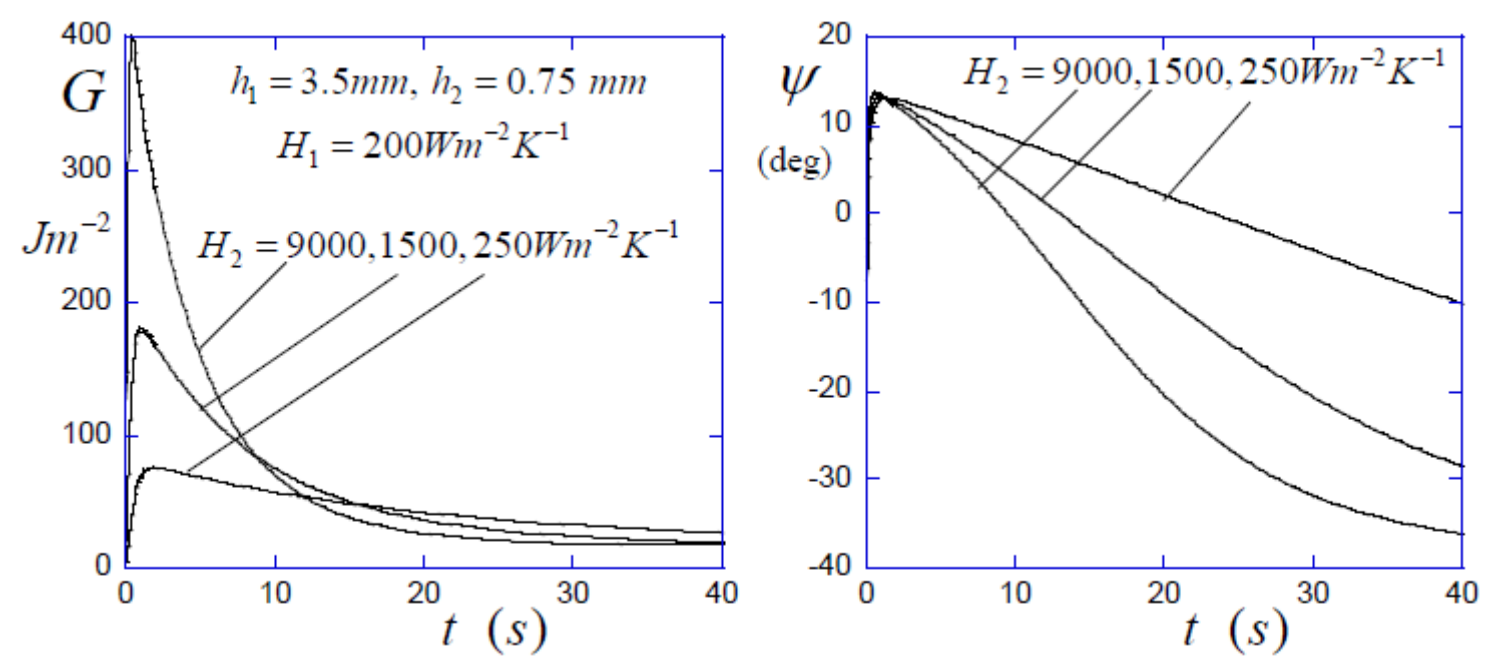

Fig. 14 Effect of the heat transfer coefficient at the coating surface, $H_{2}$, on the delamination energy release rate and mode mix for the unconstrained bilayer (8) subject to the otherwise unchanged cooling (10). The JETS reference case has $H_{1}=200 \mathrm{Wm}^{-2} \mathrm{~K}^{-1}$ and $H_{2}=1500 \mathrm{Wm}^{-2} \mathrm{~K}^{-1}$. In all cases, $T_{1}^{\text {sur }}(0)=870 \mathrm{C}$ and $T_{2}^{\text {sur }}(0)=1425 C$. 

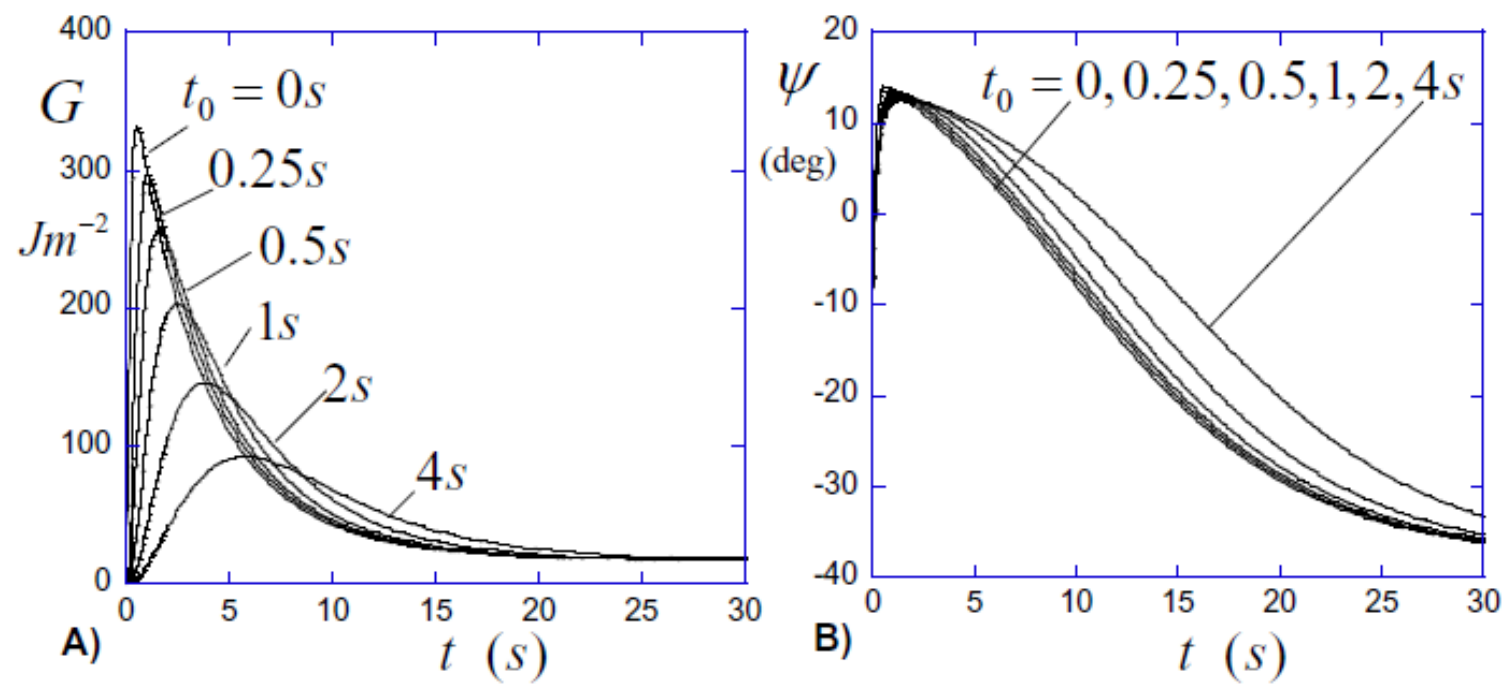

Fig. 15 The effect of the rate of switching on the cooling gas on the delamination energy release rate and mode mix as dependent on the time scale, $t_{0}$, defined in (26) for the reference unconstrained bilayer with properties (8). The heat transfer coefficients used in these simulations, $H_{1}=988 \mathrm{Wm}^{-2} \mathrm{~K}^{-1}$ and $H_{2}=4698 \mathrm{Wm}^{-2} \mathrm{~K}^{-1}$, with hot state gas temperatures, $T_{1}^{\text {gas }}(0)=38 C$ and $T_{2}^{\text {gas }}(0)=1600 C$, are consistent with the initial steadystate hot state surface temperatures, $T_{1}^{\text {sur }}(0)=870 C$ and $T_{2}^{\text {sur }}(0)=1425 C$. Starting at $t=0$ the gas temperature impinging on the coating surface is reduced to $38 \mathrm{C}$ with an exponential decay (26) characterized by $t_{0}$. The gas temperature on the substrate surface is maintained at $38 \mathrm{C}$ throughout. 


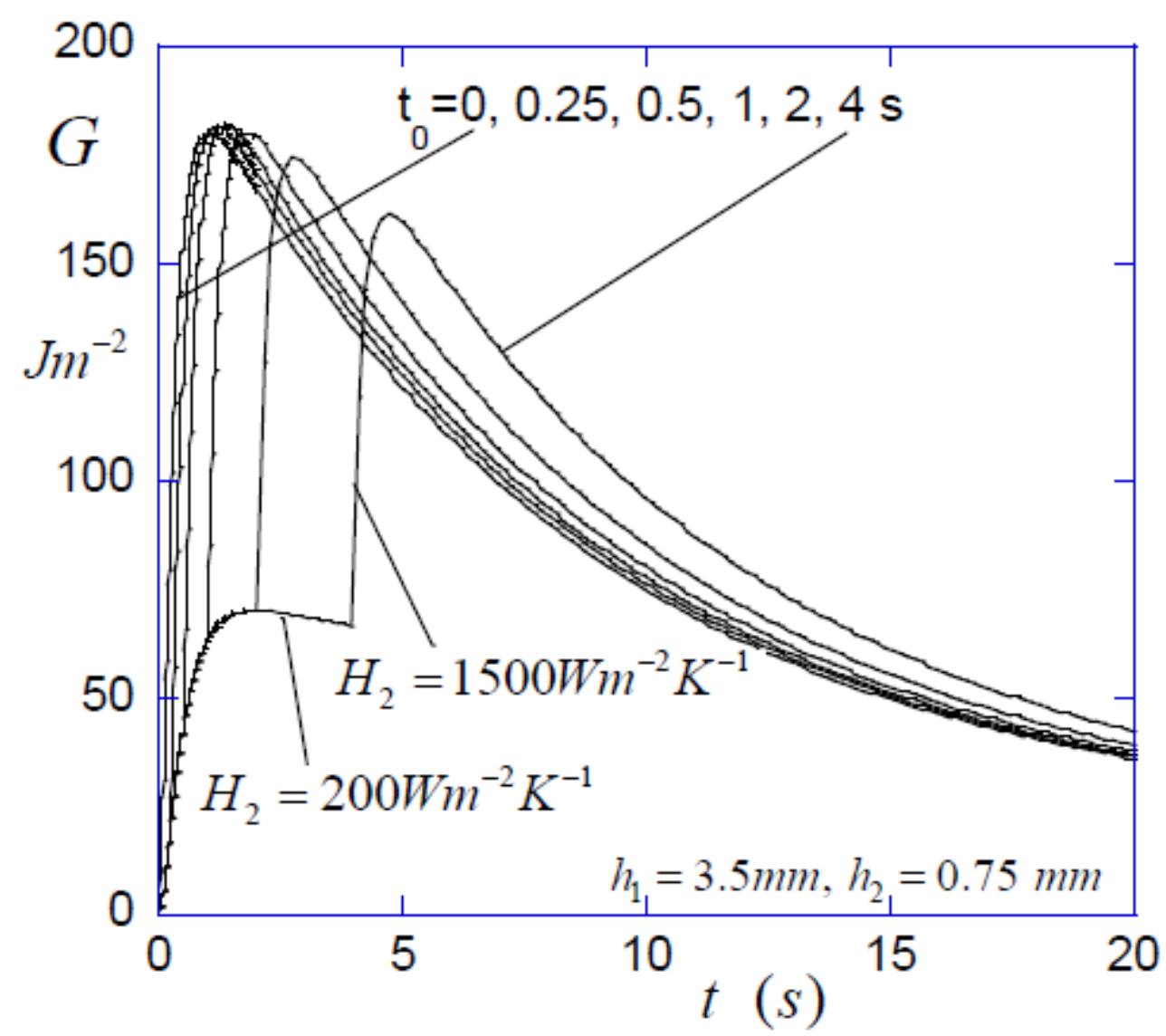

Fig. 16 The effect of a delay, $t_{0}$, in switching on a high level of the heat transfer coefficient, $\mathrm{H}_{2}$, at the coating surface on the delamination energy release rate for the reference unconstrained bilayer with properties (8). For $t \leq t_{0}, H_{2}=200 \mathrm{Wm}^{-2} \mathrm{~K}^{-1}$ and for $t>t_{0}, H_{2}=1500 \mathrm{Wm}^{-2} \mathrm{~K}^{-1} ; H_{1}=200 \mathrm{Wm}^{-2} \mathrm{~K}^{-1}$ for all $t>0$. The initial steady-state hot state temperature distribution is specified by $T_{1}^{\text {sur }}(0)=870 \mathrm{C}$ and $T_{2}^{\text {sur }}(0)=1425 \mathrm{C}$. The cooling gas temperatures, $T_{1}^{\text {gas }}=38 \mathrm{C}$ and $T_{2}^{\text {gas }}=38 \mathrm{C}$ are switched on at $t=0$. The curve for $t_{0}=0$ is the reference JETS case for unconstrained bending. 


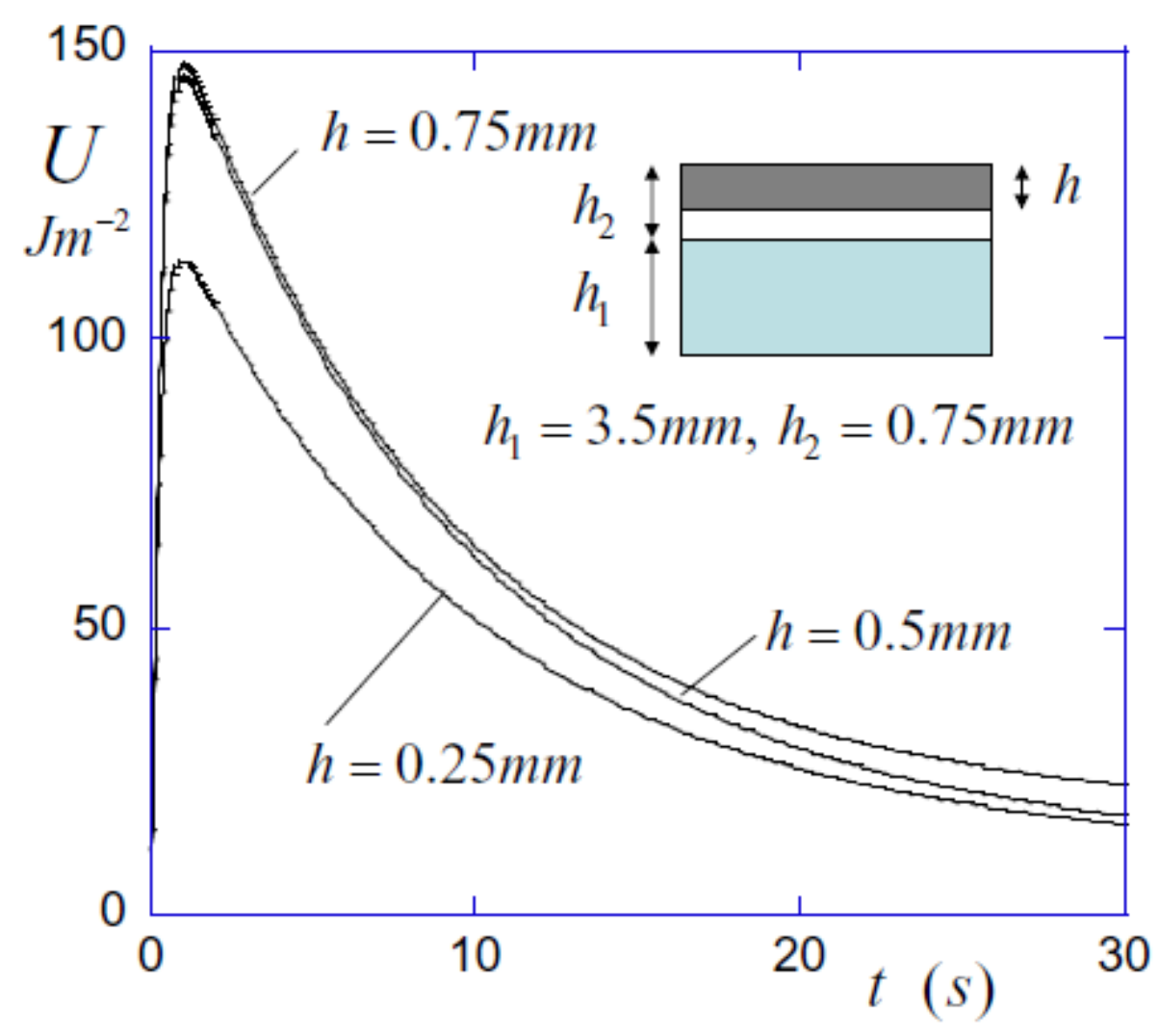

Fig. 17 The elastic energy/area, $U$, in the layer of the coating of thickness $h$ below the surface well ahead of the crack tip. This simulation is for the unconstrained reference bilayer (8) subject to the JETS scenario (9) and (10). The energy release rate for a crack within the coating propagating parallel to the interface a distance $h$ below the surface can be approximated by $U$. 